\title{
Symplectic modular symmetry in heterotic string vacua: flavor, CP, and $R$-symmetries
}

\author{
Keiya Ishiguro, ${ }^{a}$ Tatsuo Kobayashi $^{b}$ and Hajime Otsuka ${ }^{c}$ \\ ${ }^{a}$ Graduate University for Advanced Studies (Sokendai), \\ 1-1 Oho, Tsukuba, Ibaraki 305-0801, Japan \\ ${ }^{b}$ Department of Physics, Hokkaido University, \\ Sapporo 060-0810, Japan \\ ${ }^{c}$ KEK Theory Center, Institute of Particle and Nuclear Studies, KEK, \\ 1-1 Oho, Tsukuba, Ibaraki 305-0801, Japan \\ E-mail: ishigu@post.kek.jp, kobayashi@particle.sci.hokudai.ac.jp, \\ hotsuka@post.kek.jp
}

ABstract: We examine a common origin of four-dimensional flavor, CP, and $\mathrm{U}(1)_{R}$ symmetries in the context of heterotic string theory with standard embedding. We find that flavor and $\mathrm{U}(1)_{R}$ symmetries are unified into the $\mathrm{Sp}(2 h+2, \mathbb{C})$ modular symmetries of Calabi-Yau threefolds with $h$ being the number of moduli fields. Together with the $\mathbb{Z}_{2}^{\mathrm{CP}}$ CP symmetry, they are enhanced to $G \mathrm{Sp}(2 h+2, \mathbb{C}) \simeq \mathrm{Sp}(2 h+2, \mathbb{C}) \rtimes \mathbb{Z}_{2}^{\mathrm{CP}}$ generalized symplectic modular symmetry. We exemplify the $S_{3}, S_{4}, T^{\prime}, S_{9}$ non-Abelian flavor symmetries on explicit toroidal orbifolds with and without resolutions and $\mathbb{Z}_{2}, S_{4}$ flavor symmetries on three-parameter examples of Calabi-Yau threefolds. Thus, non-trivial flavor symmetries appear in not only the exact orbifold limit but also a certain class of Calabi-Yau threefolds. These flavor symmetries are further enlarged to non-Abelian discrete groups by the CP symmetry.

Keywords: Discrete Symmetries, Compactification and String Models, Superstrings and Heterotic Strings

ArXIV EPRINT: 2107.00487 


\section{Contents}

1 Introduction 1

2 Generalized symplectic modular symmetry 3

2.1 Symplectic structure of moduli fields in Calabi-Yau compactifications 3

$\begin{array}{lll}2.2 & \text { Symplectic modular flavor symmetry of matter fields } & 6\end{array}$

$\begin{array}{lll}2.3 & \mathrm{U}(1)_{R} \text { symmetry } & 9\end{array}$

$2.4 \quad$ Unification of symplectic modular flavor, $\mathrm{CP}$, and $R$-symmetries 11

3 Examples 13

3.16 D toroidal orbifolds 13

$\begin{array}{lll}3.2 & \text { Small resolutions of } T^{6} / \mathbb{Z}_{3} \text { orbifold } & 16\end{array}$

3.3 Small resolutions of $T^{6} /\left(\mathbb{Z}_{3} \times \mathbb{Z}_{3}^{\prime}\right)$ orbifold 22

3.4 Geometrical regime of blown-up $T^{6} /\left(\mathbb{Z}_{3} \times \mathbb{Z}_{3}^{\prime}\right)$ orbifold 23

3.5 Three-parameter examples of Calabi-Yau threefolds 26

$\begin{array}{lll}4 & \text { Conclusions and discussions } & 29\end{array}$

$\begin{array}{ll}\text { A Modular form of weight } 1 \text { for } T^{\prime} & 31\end{array}$

\section{Introduction}

The origin of flavor and CP symmetries is one of the unsolved and fundamental issues in the Standard Model. Understanding the underlying structure would give us a clue to find out the ultra-violet completion of the Standard Model. It was proposed in the pioneering works [1-11] that these symmetries are closely connected with the geometrical symmetry of extra-dimensional spaces. In particular, $\operatorname{SL}(2, \mathbb{Z})$ modular symmetry is of particular interest in the bottom-up approach because the non-Abelian discrete flavor symmetries, which are used to explain flavor structure of quarks and leptons such as $A_{4}, S_{4}, A_{5}$ in the past decades, are naturally embedded into the $\operatorname{SL}(2, \mathbb{Z})$, called the modular flavor symmetry [12]. The inclusion of the $\mathrm{CP}$ symmetry enlarges $\operatorname{SL}(2, \mathbb{Z})$ to the generalized modular group $\mathrm{GL}(2, \mathbb{Z})[13,14]$.

From the viewpoint of top-down approach such as the string theory, the $\operatorname{SL}(2, \mathbb{Z})$ symmetry appears in the low-energy effective action from the higher-dimensional theories on toroidal backgrounds $[15,16] .^{1}$ Furthermore, the flavor symmetry can be a quotient of $\mathrm{SL}(2, \mathbb{Z})$, and $\mathrm{CP}$ symmetry can be regarded as an outer automorphism of $\mathrm{SL}(2, \mathbb{Z})$. They can be treated in a unified manner [20]. The stabilization mechanism of moduli values providing observed masses and mixing angles of quarks/leptons is proposed in the context

\footnotetext{
${ }^{1}$ Also, the modular symmetry, in particular, its anomaly was studied in refs. [17-19].
} 
of string theory [21-23]. Hence, both flavor and CP symmetries have a common origin in the geometrical symmetries of toroidal backgrounds, and this approach would be useful to reveal the nature of extra-dimensional spaces relevant to particle physics.

In this paper, we examine the existence of modular flavor symmetry on Calabi-Yau (CY) threefolds which are promising backgrounds of the string theory. The complex structure and Kähler moduli spaces of CY threefolds are described by the symplectic structure with $\operatorname{Sp}(2 h+2, \mathbb{Z})$ symplectic groups. ${ }^{2}$ Here, $h$ is determined by the number of the complex structure moduli $h=h^{2,1}$ or the Kähler moduli $h=h^{1,1}$. Such a symplectic extension was recently discussed in refs. [23, 26-28] in both bottom-up and top-down approaches, and these flavor symmetries are called the symplectic modular flavor symmetries. Since moduli fields and matter fields are in one-to-one correspondence with each other in the context of heterotic string theory with standard embedding, the flavor symmetries of matter fields naturally lie in symplectic modular flavor symmetries. In particular, it turned out that four-dimensional (4D) CP symmetry was regarded as the outer automorphism of $\operatorname{Sp}(2 h+2, \mathbb{Z})$ on CY backgrounds [23]. These observations motivate us to examine the symplectic modular symmetry involving the flavor and CP symmetries in the context of CY symplectic moduli spaces.

The purpose of this paper is to formulate the symplectic modular flavor symmetries together with CP symmetry in the context of heterotic string theory with standard embedding and exemplify these symmetries on several toroidal and CY backgrounds. As discussed in detail in section 2, the flavor symmetries of fundamental and anti-fundamental representations of $E_{6}$ gauge group are governed by the symplectic modular symmetries in a general class of CY threefolds with large volume/complex structure, although the flavor structure depends on the intersection number of CY threefolds. Together with the results of ref. [23], we find that symplectic modular flavor symmetries are further enlarged to $G \mathrm{Sp}(2 h+2, \mathbb{Z}) \simeq \operatorname{Sp}(2 h+2, \mathbb{Z}) \rtimes \mathbb{Z}_{2}^{\mathrm{CP}}$, called "generalized symplectic modular symmetry". Furthermore, the rotation of holomorphic three-form of CY threefolds induces that of Killing spinors of $4 \mathrm{D}$ supersymmetry, namely the $\mathrm{U}(1)_{R}$ symmetry [29]. As a result, the flavor and the $\mathrm{U}(1)_{R}$ symmetries are described by $\operatorname{Sp}(2 h+2, \mathbb{C})$ symplectic modular symmetry, which results in the unification of all flavor, $\mathrm{CP}$, and $\mathrm{U}(1)_{R}$ symmetries in the context of $G \operatorname{Sp}(2 h+2, \mathbb{C})$ symmetry. We explicitly demonstrate generalized symplectic modular symmetries on toroidal orbifolds with/without resolutions and three-parameter examples of CY threefolds. It turns out that there exist $G_{\text {flavor }}=\mathbb{Z}_{2}, S_{3}, S_{4}, T^{\prime}, S_{9}$ nonAbelian flavor symmetries, and they are further enhanced into $G_{\text {flavor }} \rtimes \mathbb{Z}_{2}^{\mathrm{CP}}$ together with the CP symmetry. So far, several techniques have been developed to understand $G_{\text {flavor }}$ on toroidal orbifolds, but our approach is useful to find out the flavor structure of smooth CY threefolds, including the known toroidal orbifold regime. Indeed, our results indicate that a certain class of CY threefolds has the $S_{4}$ flavor symmetry on matter fields associated with the moduli fields.

This paper is organized as follows. In section 2, we briefly review the symplectic structure of moduli fields in CY compactifications and propose the generalized symplectic

\footnotetext{
${ }^{2}$ We refer the details about the symplectic modular symmetry to refs. [24, 25].
} 
modular symmetries of matter fields in the context of heterotic string theory with standard embedding, where fundamental and anti-fundamental representations of $E_{6}$ group are directly related to Kähler moduli and complex structure moduli, respectively. The symplectic modular flavor symmetries and their enlarged symmetries by the CP transformation are explicitly demonstrated in section 3. Section 4 is devoted to conclusions and discussions. In appendix A, we give the modular form of weight 1 for $T^{\prime}$.

\section{Generalized symplectic modular symmetry}

In section 2.1, we review the symplectic structure of moduli fields in CY compactifications with an emphasis on the large volume/complex structure regime. In section 2.2 , we give a prescription of the symplectic modular flavor symmetry of matter fields associated with the complex structure moduli and Kähler moduli in the context of heterotic string theory with standard embedding. Furthermore, the symplectic transformations of matter fields naturally incorporate the existence of $\mathrm{U}(1)_{R}$ symmetry appearing in the $4 \mathrm{D}$ effective action as shown in section 2.3. The CP transformations exchanging the fundamental and anti-fundamental representations of $E_{6}$ are regarded as the anti-holomorphic transformations of moduli fields. In section 2.4, we find that these anti-holomorphic transformations enlarge the symplectic modular symmetry into the generalized symplectic modular symmetry. As a result, the symplectic modular, flavor, $\mathrm{CP}$, and $\mathrm{U}(1)_{R}$ symmetries are treated in a unified manner.

\subsection{Symplectic structure of moduli fields in Calabi-Yau compactifications}

It was well known that the CY compactifications lead to the symplectic structure in the 4D effective action. (For more details about the symplectic structure of CY moduli spaces, see, e.g., refs. $[24,25]$.) The moduli fields associated with the CY metric are categorized into two types of moduli fields, i.e., the complex structure moduli and the Kähler moduli. Their Kähler potentials in the units of the reduced Planck mass are given by

$$
\begin{aligned}
& K_{\mathrm{cs}}=-\ln \left[-i \int_{\mathcal{M}} \Omega \wedge \bar{\Omega}\right], \\
& K_{\mathrm{ks}}=-\ln \left[\int_{\mathcal{M}} J_{c} \wedge J_{c} \wedge J_{c}\right],
\end{aligned}
$$

where $\Omega$ and $J_{c}$ denote the holomorphic three-form and (complexified) Kähler form of CY threefolds $\mathcal{M}$, respectively. Here, $J_{c}=B+i J$ consists of real Kalb-Ramond two-form $B$ and Kähler form $J$. Their moduli spaces possess the symplectic structure. For concreteness, we will focus on the complex structure moduli with large complex structure, paying attention to the symplectic transformations of the moduli fields and the prepotential in subsequent discussions. The purpose of reviewing the symplectic structure is to understand the flavor structure of matter fields in the context of heterotic string theory with standard embedding, as will be discussed in the next section. 
The holomorphic three-form $\Omega$ is expanded on the symplectic basis $\left(\alpha_{I}, \beta^{I}\right)$ of $H^{3}(\mathcal{M}, \mathbb{Z}),^{3}$

$$
\Omega=\sum_{I=0}^{h^{2,1}}\left(X^{I} \alpha_{I}-\mathcal{F}_{I} \beta^{I}\right),
$$

where $X^{I}$ are projective coordinates and the structure of $\mathcal{F}_{I}=\partial_{I} \mathcal{F}$ is determined by the prepotential $\mathcal{F}$. The orthogonal relation of the symplectic basis $\left(\alpha_{I}, \beta^{I}\right)$

$$
\int_{\mathcal{M}} \alpha_{I} \wedge \beta^{J}=\delta^{J}{ }_{I}, \quad \int_{\mathcal{M}} \beta^{J} \wedge \alpha_{I}=-\delta_{I}^{J},
$$

is invariant under the symplectic transformation:

$$
\left(\begin{array}{l}
\alpha_{I} \\
\beta^{I}
\end{array}\right) \rightarrow\left(\begin{array}{ll}
a & b \\
c & d
\end{array}\right)\left(\begin{array}{l}
\alpha_{I} \\
\beta^{I}
\end{array}\right)
$$

with

$$
\left(\begin{array}{ll}
a & b \\
c & d
\end{array}\right) \in \operatorname{Sp}\left(2 h^{2,1}+2, \mathbb{Z}\right) .
$$

Correspondingly, the vector $\left\{X^{I}, \mathcal{F}_{I}\right\}^{T}$ (the so-called period integrals of holomorphic threeform)

$$
\Pi=\left(\begin{array}{c}
\int_{A^{I}} \Omega \\
\int_{B_{I}} \Omega
\end{array}\right)=\left(\begin{array}{l}
X^{I} \\
\mathcal{F}_{I}
\end{array}\right)
$$

transforms as

$$
\left(\begin{array}{c}
X^{I} \\
\mathcal{F}_{I}
\end{array}\right) \rightarrow\left(\begin{array}{c}
\widetilde{X}^{I} \\
\widetilde{\mathcal{F}}_{I}
\end{array}\right)=\left(\begin{array}{ll}
d & c \\
b & a
\end{array}\right)\left(\begin{array}{c}
X^{I} \\
\mathcal{F}_{I}
\end{array}\right),
$$

due to the fact that the holomorphic three-form is invariant under the symplectic transformation. Here, $\widetilde{\mathcal{F}}_{I}$ is required to be a derivative of new function $\tilde{\mathcal{F}}(\tilde{X})$, namely $\widetilde{\mathcal{F}}_{I}=\partial \widetilde{\mathcal{F}}(\widetilde{X}) / \partial \widetilde{X}^{I}$, to remain within the same class of $4 \mathrm{D}$ effective action. Note that the $4 \mathrm{D} \mathcal{N}=2$ supersymmetric action is invariant under the symplectic transformation of the prepotential, $\widetilde{\mathcal{F}}(\widetilde{X})=\mathcal{F}(X)$. Furthermore, when

$$
\widetilde{\mathcal{F}}(\tilde{X})=\mathcal{F}(\tilde{X})
$$

the Lagrangian of $\mathcal{N}=2$ supergravity with $n$ Abelian vector multiplets is invariant under the symplectic transformations [30], although the prepotential itself is not invariant under them [31]. This structure holds for the system of our interest, i.e., string compactifications on $(2,2)$ superconformal field theories [32-35].

\footnotetext{
${ }^{3}$ Note that the $\operatorname{Sp}\left(2 h^{2,1}+2, \mathbb{C}\right)$ appears in the classical moduli space, but loop and quantum corrections break it to $\operatorname{Sp}\left(2 h^{2,1}+2, \mathbb{Z}\right)$, namely duality transformations of the string theory.
} 
From the symplectic transformation (2.7), it is found that $\left\{X^{I}\right\}$ takes a vector-valued modular form of $\operatorname{Sp}\left(2 h^{2,1}+2, \mathbb{Z}\right)$, namely

$$
X^{I} \rightarrow \widetilde{X}^{I}=(c \mathcal{F}+d)_{J}^{I} X^{J}=\frac{\partial \widetilde{X}^{I}}{\partial X^{J}} X^{J},
$$

and the symmetric tensor $\mathcal{F}_{I J K}=\partial_{I} \partial_{J} \partial_{K} \mathcal{F}$ obeys

$$
\mathcal{F}_{I J K} \rightarrow \widetilde{\mathcal{F}}_{I J K}=\frac{\partial X^{L}}{\partial \widetilde{X}^{I}} \frac{\partial X^{M}}{\partial \widetilde{X}^{J}} \frac{\partial X^{N}}{\partial \widetilde{X}^{K}} \mathcal{F}_{L M N},
$$

where it is required that $\partial \widetilde{X}^{I} / \partial X^{J}$ is a non-singular matrix. For instance, the invertible relation between $\widetilde{X}^{I}$ and $X^{I}$ is lost when the prepotential is a linear function of $X^{I}$ as pointed out in ref. [31]. This formula is useful to understand the symplectic transformation of Yukawa couplings among matter fields as analyzed in detail below.

In the large complex structure regime, ${ }^{4}$ the prepotential is of the explicit form

$$
\mathcal{F}(X)=\frac{1}{3 !} \kappa_{i j k} \frac{X^{i} X^{j} X^{k}}{X^{0}}=\left(X^{0}\right)^{2} F(u),
$$

where $\kappa_{i j k}$ denote the triple intersection numbers and the complex structure moduli $u^{i}$ are set by

$$
u^{i} \equiv \frac{X^{i}}{X^{0}}
$$

Here and in what follows, we still call $F(u)=\frac{1}{3 !} \kappa_{i j k} u^{i} u^{j} u^{k}$ the prepotential and take the flat coordinates

$$
X^{0}=1, \quad X^{i}=u^{i}
$$

Then, the symplectic transformation of the complex structure moduli $u^{i}$ is given by

$$
u^{i} \equiv X^{i} \rightarrow \widetilde{u}^{i}=\frac{\widetilde{X}^{i}}{\widetilde{X}^{0}}=\frac{\partial \widetilde{X}^{i}}{\partial X^{j}} \frac{X^{J}}{\widetilde{X}^{0}}=\frac{\partial \widetilde{X}^{i}}{\partial X^{j}} \frac{X^{j}}{\widetilde{X}^{0}}=\frac{\partial \widetilde{X}^{i}}{\partial X^{j}} \frac{u^{j}}{\widetilde{X}^{0}}=(c \mathcal{F}+d)_{j}^{i} \frac{u^{j}}{\widetilde{X}^{0}} .
$$

Let us discuss the symplectic transformation of Yukawa couplings $\left(F_{i j k}\right)$ among complex structure moduli. They are determined by the triple intersection numbers $\kappa_{i j k}$,

$$
F_{i j k} \equiv \frac{\partial}{\partial u^{i}} \frac{\partial}{\partial u^{j}} \frac{\partial}{\partial u^{k}} F(u)=X^{0} \mathcal{F}_{i j k}(X)=\kappa_{i j k},
$$

which correspond to the holomorphic Yukawa couplings of matter fields in the context of heterotic string theory with standard embedding. The symplectic transformation of Yukawa couplings $F_{i j k}$ can be read off from eq. (2.10), i.e.,

$$
\widetilde{F}_{i j k}=\frac{\partial}{\partial \widetilde{u}^{i}} \frac{\partial}{\partial \widetilde{u}^{j}} \frac{\partial}{\partial \widetilde{u}^{k}} \widetilde{F}(\widetilde{u})=\frac{\partial u^{l}}{\partial \widetilde{u}^{i}} \frac{\partial u^{m}}{\partial \widetilde{u}^{j}} \frac{\partial u^{n}}{\partial \widetilde{u}^{k}} F_{l m n},
$$

\footnotetext{
${ }^{4}$ Throughout this paper, we analyze the classical CY moduli space.
} 
or

$$
\widetilde{F}_{i j k}=\widetilde{X}^{0} \widetilde{\mathcal{F}}_{i j k}=\widetilde{X}^{0} \frac{\partial X^{L}}{\partial \widetilde{X}^{i}} \frac{\partial X^{M}}{\partial \widetilde{X}^{j}} \frac{\partial X^{N}}{\partial \widetilde{X}^{k}} \mathcal{F}_{L M N}=\widetilde{X}^{0} \frac{\partial X^{l}}{\partial \widetilde{X}^{i}} \frac{\partial X^{m}}{\partial \widetilde{X}^{j}} \frac{\partial X^{n}}{\partial \widetilde{X}^{k}} F_{l m n} .
$$

Hence, these two expressions lead to

$$
\frac{\partial u^{l}}{\partial \widetilde{u}^{i}}=\left(\widetilde{X}^{0}\right)^{1 / 3} \frac{\partial X^{l}}{\partial \widetilde{X}^{i}}
$$

So far, we have focused on the symplectic structure of the complex structure moduli space, but the same structure appears in the Kähler moduli space as discussed in detail in ref. [25]. When we introduce a basis of $H^{2}(\mathcal{M}, \mathbb{Z})$ as $e_{a}$, the Kähler moduli $t^{a}$ are defined by

$$
t^{a} e_{a}=B+i J=J_{c}
$$

whose prepotential in the large volume regime is given by

$$
\mathcal{G}(Y)=\frac{1}{3 !} \kappa_{a b c} \frac{Y^{a} Y^{b} Y^{c}}{Y^{0}}=\left(Y^{0}\right)^{2} G(t)
$$

with

$$
t^{a}=\frac{Y^{a}}{Y^{0}}
$$

Here, $\kappa_{a b c}$ denote the triple intersection numbers among two cycles of CY threefolds, and $\left\{Y^{A}\right\}$ represent the projective coordinates on the Kähler moduli space. In particular, $Y^{0}$ is identified with the inverse of the axio-dilaton. Analogous to the complex structure moduli, the Kähler potential (2.1) is written by

$$
\begin{aligned}
& K_{\mathrm{cs}}=-\ln \left[-i \int_{\mathcal{M}} \Omega \wedge \bar{\Omega}\right]=-\ln \left[-i\left(\bar{X}^{I} \mathcal{F}_{I}-X^{I} \overline{\mathcal{F}}_{I}\right)\right] \\
& K_{\mathrm{ks}}=-\ln \left[\int_{\mathcal{M}} J_{c} \wedge J_{c} \wedge J_{c}\right]=-\ln \left[-i\left(\bar{Y}^{A} \mathcal{G}_{A}-Y^{A} \overline{\mathcal{G}}_{A}\right)\right],
\end{aligned}
$$

with $I=0,1, \cdots, h^{2,1}, A=0,1, \cdots, h^{1,1}$, and one can deal with the symplectic structure as summarized in table 1 .

In the subsequent sections, we discuss the symplectic modular transformations of matter fields associated with the complex structure moduli and Kähler moduli. The flavor structure of matter fields can be read off from the symplectic structure of moduli Yukawa couplings.

\subsection{Symplectic modular flavor symmetry of matter fields}

In general, it is difficult to derive the flavor structure of matter fields in smooth CY compactifications due to the lack of analytical expression of the CY metric. Hence, we concentrate on the heterotic string theory with standard embedding to overcome this problem. The matter fields $27_{a}$ and $\overline{27}_{i}$ under the $E_{6}$ gauge group are in one-to-one correspondence with the Kähler moduli and the complex structure moduli, respectively. It indicates that 


\begin{tabular}{|ccc|}
\hline & Complex structure & Kähler structure \\
\hline Projective coordinates & $X^{I}$ & $Y^{A}$ \\
Moduli fields & $\left(I=1,2, \cdots, h^{2,1}+1\right)$ & $\left(A=1,2, \cdots, h^{1,1}+1\right)$ \\
& $u^{i}$ & $t^{a}$ \\
Prepotential & $\left(i=1,2, \cdots, h^{2,1}\right)$ & $\left(a=1,2, \cdots, h^{1,1}\right)$ \\
Kähler potential & $\mathcal{F}(X)=\left(X^{0}\right)^{2} F(u)$ & $\mathcal{G}(Y)=\left(Y^{0}\right)^{2} G(t)$ \\
Yukawa couplings & $-\ln \left[-i\left(\bar{X}^{I} \mathcal{F}_{I}-X^{I} \overline{\mathcal{F}}_{I}\right)\right]$ & $-\ln \left[-i\left(\bar{Y}^{A} \mathcal{G}_{A}-Y^{A} \overline{\mathcal{G}}_{A}\right)\right]$ \\
Symplectic modular group & $\partial_{i} \partial_{j} \partial_{k} F=\kappa_{i j k}$ & $\partial_{a} \partial_{b} \partial_{c} G=\kappa_{a b c}$ \\
Symplectic transformations & $u^{i} \rightarrow\left(2 h^{2,1}+2, \mathbb{Z}\right)$ & $\operatorname{Sp}\left(2 h^{1,1}+2, \mathbb{Z}\right)$ \\
& $\left(\begin{array}{ll}a & b \\
c & d\end{array}\right) \in \operatorname{Sp}\left(2 h^{2,1}+2, \mathbb{Z}\right)$ & $\left(\begin{array}{c}e f \\
g\end{array}\right) \in \operatorname{Sp}\left(2 h^{1,1}+2, \mathbb{Z}\right)$ \\
\hline
\end{tabular}

Table 1. Moduli spaces of the complex structure and the Kähler structure.

the flavor structure of matter fields is directly related to the symplectic structure in the moduli spaces. In the following discussions, we assume that the matter zero-modes appear from either the complex structure moduli sector or the Kähler moduli sector since the net number of zero-modes is determined by

$$
\chi=\frac{1}{2}\left|h^{2,1}-h^{1,1}\right|,
$$

through the Atiyah-Singer index theorem. We will focus on the matter fields associated with either the complex structure moduli or the Kähler moduli in order to reveal the relation between the flavor symmetry of matter fields and symplectic modular symmetry appearing in the moduli effective action.

It was known that the matter Kähler metric is of the form ${ }^{5}$

$$
\begin{aligned}
& K_{a \bar{b}}^{(27)}=e^{\frac{1}{3}\left(K_{\mathrm{cs}}-K_{\mathrm{ks}}\right)}\left(K_{\mathrm{ks}}\right)_{a \bar{b}}, \\
& K_{i \bar{j}}^{(2 \overline{7})}=e^{-\frac{1}{3}\left(K_{\mathrm{cs}}-K_{\mathrm{ks}}\right)}\left(K_{\mathrm{cs}}\right)_{i \bar{j}},
\end{aligned}
$$

where $\left(K_{\mathrm{cs}}\right)_{i \bar{j}}=\partial_{i} \partial_{\bar{j}} K_{\mathrm{cs}},\left(K_{\mathrm{ks}}\right)_{a \bar{b}}=\partial_{a} \partial_{\bar{b}} K_{\mathrm{ks}}, A^{i}$ and $A^{a}$ denote the matter chiral superfields, respectively. ${ }^{6}$ At the moment, we study the relation between $\overline{27}_{i}$ matter fields and the complex structure moduli $u^{i}$ for concreteness. The Kähler potential of the complex structure moduli in the large complex structure regime

$$
K_{\mathrm{cS}}=-\ln \left[-i \int_{\mathcal{M}} \Omega \wedge \bar{\Omega}\right]=-\ln \left[-i\left|X^{0}\right|^{2} \frac{\kappa_{i j k}}{6}\left(u^{i}-\bar{u}^{i}\right)\left(u^{j}-\bar{u}^{j}\right)\left(u^{k}-\bar{u}^{k}\right)\right],
$$

\footnotetext{
${ }^{5}$ The overall factor $e^{ \pm \frac{1}{3}\left(K_{\mathrm{cs}}-K_{\mathrm{ks}}\right)}$ takes the different form on toroidal orbifolds in the example of section 3 due to the enlarged symmetry [36].

${ }^{6}$ In the following, we call matter chiral superfields as matters for simplicity.
} 
indicates that the matter Kähler metric obeys

$$
K_{i \bar{j}}^{(\overline{27})} \propto\left|X^{0}\right|^{2 / 3}\left(K_{\mathrm{cS}}\right)_{i \bar{j}}
$$

up to the factor relevant to the Kähler moduli. Hence, symplectic transformations of $\overline{27}_{i}$ matter fields are almost the same with those of the moduli fields $u^{i}$, up to the $X^{0}$ factor. Indeed, the matter Kähler metric transforms under the symplectic modular transformations as

$$
K_{i \bar{j}}^{(\overline{27})} \rightarrow\left|\widetilde{X}^{0}\right|^{2 / 3}\left(K_{\mathrm{cs}}\right) \widetilde{{ }^{m}} \frac{\partial \widetilde{u}^{i}}{\partial u^{l}} \frac{\partial \widetilde{\bar{u}}^{j}}{\partial \bar{u}^{m}}
$$

up to the factor $e^{K_{\mathrm{ks}} / 3}$. It turns out that the Kähler potential of $\overline{27}_{i}$ matter fields has a modular invariance under the following symplectic transformation of matter fields:

$$
A^{i} \rightarrow \widetilde{A}^{i}=\left(\widetilde{X}^{0}\right)^{1 / 3} \frac{\partial \widetilde{u}^{i}}{\partial u^{l}} A^{l}=\frac{\partial \widetilde{X}^{i}}{\partial X^{j}} A^{j},
$$

where we use eq. (2.18). Since the matrix

$$
\frac{\partial \widetilde{X}^{i}}{\partial X^{j}}=(c \mathcal{F}+d)_{j}^{i}
$$

is an element of the subgroup of $\operatorname{Sp}\left(2 h^{2,1}+2, \mathbb{Z}\right)$, the flavor structure of matter fields is governed by the symplectic structure. In what follows, we adopt the gauge $X^{0}=1$ unless we specify it. As will be discussed later, the above modular transformation is indeed consistent with the Kähler invariance of the action by checking the Kähler invariant quantity.

On the other hand, the matter superpotential takes the form

$$
W=F_{i j k} A^{i} A^{j} A^{k}
$$

where the matter Yukawa couplings are the same as the Yukawa couplings of the moduli fields. As we derived before, the $\operatorname{Sp}\left(2 h^{2,1}+2, \mathbb{Z}\right)$ modular transformations of their Yukawa couplings are provided by

$$
\widetilde{F}_{i j k}=\widetilde{X}^{0} \frac{\partial X^{l}}{\partial \widetilde{X}^{i}} \frac{\partial X^{m}}{\partial \widetilde{X}^{j}} \frac{\partial X^{n}}{\partial \widetilde{X}^{k}} F_{l m n} .
$$

Hence, we arrive at the symplectic modular transformation of the Kähler potential and the superpotential:

$$
K_{\mathrm{cs}} \rightarrow K_{\mathrm{cs}}-\ln \left|\tilde{X}^{0}\right|^{2}, \quad W \rightarrow \tilde{X}^{0} W,
$$

from which the Kähler invariant quantity

$$
e^{K}|W|^{2}
$$

is invariant under the symplectic modular transformation. 


\begin{tabular}{|ccc|}
\hline & $\begin{array}{c}\overline{27} \text { matter fields } \\
\text { (Complex structure) }\end{array}$ & $\begin{array}{c}27 \text { matter fields } \\
\text { (Kähler structure) }\end{array}$ \\
\hline Matter fields & $A^{i}$ & $A^{a}$ \\
Moduli fields & $u^{i}$ & $t^{a}$ \\
Superpotential & $F_{i j k} A^{i} A^{j} A^{k}$ & $G_{a b c} A^{a} A^{b} A^{c}$ \\
Symplectic transformations & $A^{i} \rightarrow \widetilde{A}^{i}=\frac{\partial \widetilde{X}^{i}}{\partial X^{j}} A^{j}$ & $A^{a} \rightarrow \widetilde{A}^{a}=\frac{\partial \widetilde{Y}^{a}}{\partial Y^{b}} A^{b}$ \\
& $u^{i} \rightarrow \widetilde{u}^{i}=\left(\widetilde{X}^{0}\right)^{-1} \frac{\partial \widetilde{X}^{i}}{\partial X^{j}} u^{j}$ & $t^{a} \rightarrow \widetilde{t}^{a}=\left(\widetilde{Y}^{0}\right)^{-1} \frac{\partial \widetilde{Y}^{a}}{\partial Y^{b}} t^{b}$ \\
& $F_{i j k} \rightarrow \widetilde{F}_{i j k}=\widetilde{X}^{0} \frac{\partial X^{l}}{\partial \widetilde{X}^{i}} \frac{\partial X^{m}}{\partial \widetilde{X}^{j}} \frac{\partial X^{n}}{\partial \widetilde{X}^{k}} F_{l m n}$ & $G_{a b c} \rightarrow \widetilde{G}_{a b c}=\widetilde{Y}^{0} \frac{\partial Y^{d}}{\partial \widetilde{Y}^{a}} \frac{\partial Y^{e}}{\partial \widetilde{Y}^{b}} \frac{\partial Y^{f}}{\partial \widetilde{Y}^{c}} G_{d e f}$ \\
Flavor symmetry & $\frac{\partial \widetilde{X}^{i}}{\partial X^{j}}=(c \mathcal{F}+d)_{j}^{i}$ & $\frac{\partial \widetilde{Y}^{a}}{\partial Y^{b}}=(g \mathcal{G}+h)_{b}^{a}$ \\
& $G_{\text {flavor }} \subset \operatorname{Sp}\left(2 h^{2,1}+2, \mathbb{Z}\right)$ & $G_{\text {flavor }} \subset \operatorname{Sp}\left(2 h^{1,1}+2, \mathbb{Z}\right)$ \\
\hline
\end{tabular}

Table 2. Symplectic modular flavor symmetries.

We conclude that the flavor symmetry of matter fields $\overline{27}_{i}, G_{\text {flavor }}$, belongs to the $\operatorname{Sp}\left(2 h^{2,1}+2, \mathbb{Z}\right)$ modular symmetry,

$$
G_{\text {flavor }} \subset \operatorname{Sp}\left(2 h^{2,1}+2, \mathbb{Z}\right),
$$

and the flavor structure is governed by the symplectic structure of the complex structure moduli. The same statements also hold for the $27_{a}$ matters associated with the Kähler moduli, whose prepotential is given in eq. (2.20) in the large volume regime. The flavor structure of $27_{a}$ matters is determined by the holomorphic Yukawa coupling $\kappa_{a b c}$. For that reason, we call the flavor symmetry of matter fields the symplectic modular flavor symmetry. The symplectic transformations of matter zero-modes, moduli, and holomorphic Yukawa couplings are summarized in table 2. It is a natural generalization of $\operatorname{Sp}(2, \mathbb{Z}) \simeq$ $\mathrm{SL}(2, \mathbb{Z})$ discussed in the toroidal background [1-11]. Since it is difficult to derive the flavor symmetries in a background-independent way, we explicitly demonstrate the existence of symplectic modular flavor symmetries on toroidal orbifolds with and without resolutions and some classes of CY threefolds in section 3.

\section{$2.3 \mathrm{U}(1)_{R}$ symmetry}

In addition to the symplectic modular flavor symmetry, there exists a $\mathrm{U}(1)_{R}$ symmetry in the $4 \mathrm{D} \mathcal{N}=1$ supersymmetric effective action. From the cubic matter superpotential (2.30), one can assign the $R$-charge $2 / 3$ for the matter fields in a conventional way due to the fact that the superpotential has $R$-charge 2 . Here, we examine the $\overline{27}_{i}$ matter fields, but $27_{a}$ matter fields also have $R$-charge $2 / 3$ due to the same cubic superpotential. In this section, we discuss the relation between the symplectic modular symmetry and $\mathrm{U}(1)_{R}$ symmetry.

Let us consider the symplectic transformations in the basis where the Yukawa couplings do not have the factor $\tilde{X}^{0}$,

$$
\begin{gathered}
A^{i} \rightarrow \widetilde{A}^{i}=\left(\widetilde{X}^{0}\right)^{1 / 3} \frac{\partial \widetilde{u}^{i}}{\partial u^{l}} A^{l}, \\
F_{i j k} \rightarrow \widetilde{F}_{i j k}=\frac{\partial u u^{l}}{\partial \widetilde{u}^{i}} \frac{\partial u^{m}}{\partial \widetilde{u}^{j}} \frac{\partial u^{n}}{\partial \widetilde{u}^{k}} F_{l m n} .
\end{gathered}
$$


Since the $\mathrm{U}(1)_{R}$ symmetry is not the flavor symmetry, the $\left(\widetilde{X}^{0}\right)^{1 / 3}$ factor appearing in the symplectic transformation of matter fields would play a role of $R$-symmetric transformation. In this respect, let us set

$$
\widetilde{X}^{0}=e^{2 i \alpha},
$$

with $\alpha$ being a constant relevant to the $R$-symmetric transformation [37]. This assignment is consistent with the symplectic modular symmetry and it gives rise to the $R$-charge 2 for the moduli fields. Indeed, the $R$-symmetric transformations for the period integrals in the large complex structure regime correspond to

$$
\left(\begin{array}{c}
X^{I} \\
\mathcal{F}_{I}
\end{array}\right) \rightarrow\left(\begin{array}{c}
\tilde{X}^{I} \\
\widetilde{\mathcal{F}}_{I}
\end{array}\right)=\widetilde{X}^{0}\left(\begin{array}{c}
X^{I} \\
\mathcal{F}_{I}
\end{array}\right),
$$

with

$$
\begin{aligned}
& \left\{X^{0}, X^{i}, \mathcal{F}_{0}, \mathcal{F}_{i}\right\}=\left\{1, u^{i},-\frac{1}{3 !} \kappa_{i j k} u^{i} u^{j} u^{k}, \frac{1}{2} \kappa_{i j k} u^{j} u^{k}\right\}, \\
& \left\{\widetilde{X}^{0}, \widetilde{X}^{i}, \widetilde{\mathcal{F}}_{0}, \widetilde{\mathcal{F}}_{i}\right\}=\widetilde{X}^{0}\left\{1, \widetilde{u}^{i},-\frac{1}{3 !} \kappa_{i j k} \widetilde{u}^{i} \widetilde{u}^{j} \widetilde{u}^{k}, \frac{1}{2} \kappa_{i j k} \widetilde{u}^{j} \widetilde{u}^{k}\right\} .
\end{aligned}
$$

As stated in ref. [29], $R$-symmetric transformation corresponds to the holomorphic transformation $f$ mapping the holomorphic three-form of CY threefolds $\Omega$ into a multiple of itself,

$$
f \cdot \Omega=e^{i \gamma} \Omega
$$

with $\gamma$ being the constant. Recalling that the $\Omega$ is constructed by a covariantly constant positive-chirality spinor $\eta$, namely $\Omega_{i j k}=\eta^{T} \Gamma_{i j k} \eta$ with three products of Gamma matrix $\Gamma_{i j k}$, the holomorphic mapping $f$ rotates the $\eta$,

$$
f \cdot \eta=e^{i \gamma / 2} \eta
$$

namely the $4 \mathrm{D} \mathcal{N}=1$ supersymmetry by a phase of $e^{i \gamma / 2}$ for positive chirality spinor. The negative chirality spinor is rotated by an amount of $e^{-i \gamma / 2}$. In this way, the phase shift of $\Omega$ gives rise to the $R$-symmetry in $4 \mathrm{D} \mathcal{N}=1$ supersymmetric theory. In our setup, our finding $R$-symmetric transformation $\tilde{X}^{0}=e^{2 i \alpha}$ corresponds to $\gamma=2 \alpha$. Note that the existence of $\mathrm{U}(1)_{R}$ symmetry is related to the discrete $\mathbb{Z}_{3}^{R} R$-symmetry appearing in the matter superpotential,

$$
W=F_{i j k} A^{i} A^{j} A^{k},
$$

by assigning the charge 1 for all the matter fields. When we focus on the overall complex structure modulus $u^{1}=u^{2}=\cdots=u^{h^{2,1}}$, the action is invariant under the overall $\mathrm{SL}(2, \mathbb{Z})_{\text {overall }}$ symmetry. From the fact that the superpotential has modular weight -3 under $\operatorname{SL}(2, \mathbb{Z})_{\text {overall }}$, the matter fields have charge -1 . That would be related to the origin of charge 1 under the discrete $\mathbb{Z}_{3}^{R} R$-symmetry. ${ }^{7}$ Similar statements also hold for the Kähler moduli space, by identifying $Y^{0}$ with the $R$-symmetric transformations of $27_{a}$ matter

\footnotetext{
${ }^{7}$ The relations between $R$-symmetry and the modular symmetry, in particular, their anomalies were studied in refs. [38, 39].
} 
fields. Hence, $R$-symmetric transformations would be related to the phase rotation of the axio-dilaton. It is notable that the Kähler form consists of Killing spinors, $J_{m \bar{n}}=i \eta^{\dagger} \Gamma_{m \bar{n}} \eta$ with two products of Gamma matrix $\Gamma_{m \bar{n}}$ in the complex basis $z^{m}$ with $m=1,2,3$, but it does not change under the $R$-transformation.

As a result, $\mathrm{U}(1)_{R}$ symmetry is also unified in the context of symplectic modular symmetry, i.e.,

$$
\operatorname{Sp}(2 h+2, \mathbb{C}) \supset \operatorname{Sp}(2 h+2, \mathbb{Z}) \times \mathrm{U}(1)_{R}
$$

with $h=h^{2,1}$ or $h^{1,1}$.

\subsection{Unification of symplectic modular flavor, CP, and $R$-symmetries}

Following refs. [40-42], one can identify the 4D CP symmetry with the simultaneous transformation of $4 \mathrm{D}$ parity and the $6 \mathrm{D}$ orientation reversing. They belong to the $10 \mathrm{D}$ proper Lorentz transformation. We recall that the 10D Majorana-Weyl spinor 16 decomposes under the 10D Lorentz symmetry $\mathrm{SO}(1,9)=\mathrm{SO}(1,3) \times \mathrm{SO}(6)$,

$$
16=\left(2_{L}, 4_{+}\right) \oplus\left(2_{R}, \overline{4}_{-}\right)
$$

where $2_{L}$ and $2_{R}$ denote the left- and right-handed spinors of SL(2, $\left.\mathbb{C}\right)$, and $4_{+}, \overline{4}_{-}$represent the positive and negative chirality spinors of $\mathrm{SU}(4) \simeq \mathrm{SO}(6)$, respectively. Hence, the $4 \mathrm{D}$ parity and $6 \mathrm{D}$ orientation reversing exchanges $\left(2_{L}, 4_{+}\right)$into $\left(2_{R}, \overline{4}_{-}\right)$which is in one-to-one correspondence with the $4 \mathrm{D} \mathrm{CP}$ transformation of $E_{6}$ matter fields: $27 \rightarrow \overline{27}$. It suggests that the $4 \mathrm{D} \mathrm{CP}$ transformation is identified with the geometrical symmetry of the internal manifold.

The $6 \mathrm{D}$ orientation reversing changes the sign of the volume form of $\mathrm{CY}$ threefolds. In the local coordinate of CY threefolds $z^{m}$ with $m=1,2,3$, the following transformation

$$
z^{m} \rightarrow-\bar{z}^{m}
$$

changes the sign of the volume form. ${ }^{8}$ To see the orientation reversing of the volume form, we examine the transformation of the holomorphic three-form $\Omega$ and Kähler form $J$. Eq. (2.44) gives rise to the $\mathbb{Z}_{2}^{\mathrm{CP}}$ transformation:

$$
\begin{array}{r}
\Omega \rightarrow-\bar{\Omega} \\
J_{c} \rightarrow \overline{J_{c}}
\end{array}
$$

where the anti-holomorphic transformation of $\Omega$ is consistent with its local expansion $\Omega=$ $d z^{1} \wedge d z^{2} \wedge d z^{3}$. On CY threefolds, the $6 \mathrm{D}$ volume form $d V$ is expressed by the $\Omega$,

$$
i \Omega \wedge \bar{\Omega}=|\Omega|^{2} d V
$$

thereby the transformation (2.45) changes the sign of the volume form, namely the $6 \mathrm{D}$ orientation reversing.

\footnotetext{
${ }^{8}$ The minus sign is a matter of convention.
} 
We comment on the orientation reversing of the Kähler form $J_{c}$ which is locally given by $J_{c}=B+i J=\left(b_{m \bar{n}}+i g_{m \bar{n}}\right) d z^{m} \wedge d \bar{z}^{\bar{n}}$, respectively. Recall that the real part of complexified Kähler form $\left(b_{m \bar{n}}\right)$ originates from the Kalb-Ramond field, leading to the pseudo scalars (axions) in the 4D effective action through the hodge duality. Since the axions are 4D CP-odd fields through the $4 \mathrm{D}$ Chern-Simons couplings, $b_{m \bar{n}}$ is required to be transformed as $b_{m \bar{n}} \rightarrow-b_{m \bar{n}}$. By contrast, the imaginary part of complexified Kähler form $\left(g_{m \bar{n}}\right)$ is a $\mathrm{CP}$-even field. Hence, we arrive at the CP transformation of the complexified Kähler form as in eq. (2.45).

As discussed in ref. [43], the anti-holomorphic transformation of the holomorphic threeform induces the anti-holomorphic transformation of the complex structure moduli, and the CP-transformation of the complexified Kähler form also induces the anti-holomorphic transformation of the Kähler moduli due to the fact that $b_{m \bar{n}}$ and $g_{m \bar{n}}$ are CP-odd and -even fields, respectively. Then, we arrive at the CP transformation of the moduli fields:

$$
u^{i} \rightarrow-\bar{u}^{i}, \quad t^{a} \rightarrow-\bar{t}^{a},
$$

where we take the convention with $\operatorname{Im}\left(u^{i}\right)>0$ and $\operatorname{Im}\left(t^{a}\right)>0$. Furthermore, since the real part of the axio-dilaton $Y^{0}$ originating from the Kalb-Ramond six-form is the universal axion, the CP transformation gives rise to

$$
Y^{0} \rightarrow-\bar{Y}^{0}
$$

It is trivial to check that these anti-holomorphic transformations are not the element of symplectic modular groups, but they belong to the generalized symplectic modular group $G \mathrm{Sp}(2 g+2, \mathbb{Z}) \simeq \mathrm{Sp}(2 g+2, \mathbb{Z}) \rtimes \mathbb{Z}_{2}^{\mathrm{CP}}$ with $g=h^{2,1}, h^{1,1}$. Indeed, the $\mathbb{Z}_{2}^{\mathrm{CP}}$ transformation acts on the projective coordinates in the complex structure and the Kähler structure moduli spaces ${ }^{9}$

$$
\left(\begin{array}{c}
X^{0} \\
X^{i} \\
\mathcal{F}_{0} \\
\mathcal{F}_{i}
\end{array}\right) \rightarrow-\left(\begin{array}{c}
\bar{X}^{0} \\
-\bar{X}^{i} \\
-\overline{\mathcal{F}}_{0} \\
\overline{\mathcal{F}}_{i}
\end{array}\right)=\mathcal{C P}\left(\begin{array}{c}
X^{0} \\
X^{i} \\
\mathcal{F}_{0} \\
\mathcal{F}_{i}
\end{array}\right), \quad\left(\begin{array}{c}
Y^{0} \\
Y^{a} \\
\mathcal{G}_{0} \\
\mathcal{G}_{a}
\end{array}\right) \rightarrow-\left(\begin{array}{c}
\bar{Y}^{0} \\
-\bar{Y}^{a} \\
-\overline{\mathcal{G}}_{0} \\
\overline{\mathcal{G}}_{a}
\end{array}\right)=\mathcal{C P}\left(\begin{array}{c}
Y^{0} \\
Y^{a} \\
\mathcal{G}_{0} \\
\mathcal{G}_{a}
\end{array}\right)
$$

with

$$
\mathcal{C P}=\left(\begin{array}{cccc}
-1 & 0 & 0 & 0 \\
0 & \mathbf{1} & 0 & 0 \\
0 & 0 & 1 & 0 \\
0 & 0 & 0 & -\mathbf{1}
\end{array}\right) \notin \operatorname{Sp}\left(2 h^{2,1}+2, \mathbb{Z}\right), \operatorname{Sp}\left(2 h^{1,1}+2, \mathbb{Z}\right)
$$

Here, we use the shorthand notation for the identity matrix $\mathbf{1}$, which is rank $h^{2,1}$ for the complex structure moduli and $h^{1,1}$ for the Kähler moduli, respectively. Hence, they do not belong to the element of $\operatorname{Sp}\left(2 h^{2,1}+2, \mathbb{Z}\right)$ and $\operatorname{Sp}\left(2 h^{1,1}+2, \mathbb{Z}\right)$, due to the fact that the CP-transformation does not have the symplectic structure $\mathcal{C P} \mathcal{P}^{T} \cdot \Sigma \cdot \mathcal{C P}=\Sigma^{T} \neq \Sigma$. Rather, the CP transformation is regarded as an outer automorphism of the symplectic

\footnotetext{
${ }^{9}$ Here, we adopt the minus sign for the CP transformation in the complex structure moduli space, although the positive sign is allowed.
} 
modular group, i.e., $\mathcal{C P} \cdot \gamma \cdot \mathcal{C P}{ }^{-1}$ with $\gamma$ being the transformation of the $\operatorname{Sp}\left(2 h^{2,1}+2, \mathbb{Z}\right)$ or $\operatorname{Sp}\left(2 h^{1,1}+2, \mathbb{Z}\right)$ modular group [23]..$^{10}$

As a result, the symplectic modular group together with the CP transformation is enlarged to the generalized symplectic modular group. In particular, the flavor symmetry belonging to the symplectic modular symmetry is also enhanced to the non-Abelian symmetry,

$$
\begin{aligned}
& G \mathrm{Sp}\left(2 h^{2,1}+2, \mathbb{Z}\right) \supset G_{\text {flavor }} \rtimes \mathbb{Z}_{2}^{\mathrm{CP}}, \\
& G \mathrm{Sp}\left(2 h^{1,1}+2, \mathbb{Z}\right) \supset G_{\text {flavor }} \rtimes \mathbb{Z}_{2}^{\mathrm{CP}},
\end{aligned}
$$

as exemplified in the next section. The $\mathrm{U}(1)_{R}$ symmetry further enhances them into

$$
\begin{aligned}
& G \mathrm{Sp}\left(2 h^{2,1}+2, \mathbb{C}\right) \supset G_{\text {flavor }} \rtimes \mathbb{Z}_{2}^{\mathrm{CP}} \times \mathrm{U}(1)_{R}, \\
& G \mathrm{Sp}\left(2 h^{1,1}+2, \mathbb{C}\right) \supset G_{\text {flavor }} \rtimes \mathbb{Z}_{2}^{\mathrm{CP}} \times \mathrm{U}(1)_{R} .
\end{aligned}
$$

In the following analysis, we explicitly demonstrate the symplectic modular flavor symmetry on several backgrounds. This approach would be deserved to be the ultra-violet completion of the phenomenological model buildings in the bottom-up approach, where the symplectic modular flavor symmetry is used to explain the flavor structure of quarks and leptons.

\section{Examples}

In this section, we show the existence of generalized modular symmetry involving the flavor and CP symmetries on several backgrounds. Starting from the simplest 6D toroidal orbifolds in section 3.1, we deal with several blown-up toroidal orbifolds, where the size of blow-up radii is considered small enough in sections 3.2 and 3.3 and large enough in section 3.4. The three-parameter examples of CY threefolds are analyzed in section 3.5.

\section{1 $6 \mathrm{D}$ toroidal orbifolds}

We begin with toroidal orbifolds with an emphasis on the flavor symmetry of untwisted modes. The twisted (blow-up) modes are analyzed in detail in the next sections. The simplest but non-trivial examples realizing the flavor symmetries of untwisted modes are based on $T^{6} / \mathbb{Z}_{N}$ and $T^{6} /\left(\mathbb{Z}_{N} \times \mathbb{Z}_{M}\right)$ with $h_{\text {untw }}^{1,1}=3$, whose prepotential is given by ${ }^{11}$

$$
G=t^{1} t^{2} t^{3}
$$

leading to the Kähler potential [36]

$$
K_{\mathrm{ks}}=-\ln \left[i\left(t^{1}-\bar{t}^{1}\right)\left(t^{2}-\bar{t}^{2}\right)\left(t^{3}-\bar{t}^{3}\right)-\sum_{a}\left|A^{a}\right|^{2}\right]-\ln \left|Y^{0}\right|^{2} .
$$

Here, we include $Y^{0}$ for the later purpose, and the matter fields are assumed to be expanded around $\left|A^{a}\right| \ll 1$. Correspondingly, the matter superpotential is of the form

$$
W=Y^{0} A^{1} A^{2} A^{3} .
$$

\footnotetext{
${ }^{10}$ Outer automorphisms of flavor symmetries were studied as generalized CP symmetries [44-46].

${ }^{11}$ The following discussions are applicable to the sector of $h^{2,1}=3$ complex structure moduli sector as well.
} 
Obviously, there appear the $\Pi_{a=1}^{3} \mathrm{SL}(2, \mathbb{Z})_{a}$ symmetries generated by

$$
S^{(a)}: t^{a} \rightarrow-1 / t^{a}, \quad T^{(a)}: t^{a} \rightarrow t^{a}+1,
$$

for $\Pi_{a=1}^{3} \mathrm{SL}(2, \mathbb{Z})_{a}$. They originate from factorizable three tori: $T^{6}=\left(T^{2}\right)^{3}$. In addition, we find that there exists a $S_{4}$ symmetry by assigning $\left\{t^{1}, t^{2}, t^{3}\right\}$ for $S_{4}$ triplet and $Y^{0}$ for $S_{4}$ singlet. Although the $S_{4}$ group has two triplets $\left\{\mathbf{3}, \mathbf{3}^{\prime}\right\}$ and two singlets $\left\{\mathbf{1}, \mathbf{1}^{\prime}\right\}$, the superpotential is invariant under two cases: (i) $\left\{t^{1}, t^{2}, t^{3}\right\}$ for $\mathbf{3}$ and $Y^{0}$ for $\mathbf{1}$, (ii) $\left\{t^{1}, t^{2}, t^{3}\right\}$ for $\mathbf{3}^{\prime}$ and $Y^{0}$ for $\mathbf{1}^{\prime}$. (See for non-Abelian discrete symmetries and notation of representation, refs. [47, 48]. ) When we take the gauge $Y^{0}=1$, namely $S_{4}$ trivial singlet $\mathbf{1}$, the transformation of the $S_{4}$ triplet $\left\{t^{1}, t^{2}, t^{3}\right\}$ is given by

$$
\begin{aligned}
& P: t^{1} \rightarrow t^{3}, \quad t^{2} \rightarrow t^{1}, \quad t^{3} \rightarrow t^{2}, \\
& Q: t^{1} \rightarrow-t^{1}, \quad t^{2} \rightarrow-t^{3}, \quad t^{3} \rightarrow t^{2},
\end{aligned}
$$

where $P$ corresponds to $S_{3}$ permutation group of three $\mathrm{SL}(2, \mathbb{Z})_{a}$ [49]. In the context of heterotic string theory with standard embedding, these symmetries are identified with the flavor symmetries of matter fields. Indeed, the matter fields $\left\{A^{1}, A^{2}, A^{3}\right\}$ are also identified with the $S_{4}$ triplet representation under which the effective action of matter fields is invariant.

Our purpose in this section is to check whether these symmetries together with the $\mathrm{CP}$ transformation can be embedded into the $G \operatorname{Sp}(8, \mathbb{Z})$ generalized symplectic modular symmetry. The symplectic structure is understood from the symplectic transformation of the period vector

$$
\left(\begin{array}{c}
Y^{A} \\
\mathcal{G}_{A}
\end{array}\right)=\left(\begin{array}{c}
Y^{0} \\
Y^{1} \\
Y^{2} \\
Y^{3} \\
\mathcal{G}_{0} \\
\mathcal{G}_{1} \\
\mathcal{G}_{2} \\
\mathcal{G}_{3}
\end{array}\right)=\left(\begin{array}{c}
Y^{0} \\
Y^{1} \\
Y^{2} \\
Y^{3} \\
-Y^{1} Y^{2} Y^{3} /\left(Y^{0}\right)^{2} \\
Y^{2} Y^{3} / Y^{0} \\
Y^{1} Y^{3} / Y^{0} \\
Y^{1} Y^{2} / Y^{0}
\end{array}\right)=Y^{0}\left(\begin{array}{c}
1 \\
t^{1} \\
t^{2} \\
t^{3} \\
-t^{1} t^{2} t^{3} \\
t^{2} t^{3} \\
t^{1} t^{3} \\
t^{1} t^{2}
\end{array}\right)
$$

leading to the Kähler potential (3.2). Since the period vector is a fundamental representation of $\operatorname{Sp}(8, \mathbb{Z})$, the symplectic transformation is represented by

$$
\left(\begin{array}{c}
Y^{A} \\
\mathcal{G}_{A}
\end{array}\right)=Y^{0}\left(\begin{array}{c}
1 \\
t^{1} \\
t^{2} \\
t^{3} \\
-t^{1} t^{2} t^{3} \\
t^{2} t^{3} \\
t^{1} t^{3} \\
t^{1} t^{2}
\end{array}\right) \rightarrow \widetilde{Y}^{0}\left(\begin{array}{c}
1 \\
\widetilde{t}^{1} \\
\widetilde{t}^{2} \\
\widetilde{t}^{3} \\
-\widetilde{t}^{1} \widetilde{t}^{2} \widetilde{t}^{3} \\
\widetilde{t}^{2} \widetilde{t}^{3} \\
\widetilde{t}^{1} \widetilde{t}^{3} \\
\widetilde{t}^{1} \widetilde{t}^{2}
\end{array}\right)=Y^{0} \times \mathcal{R}\left(\begin{array}{c}
1 \\
t^{1} \\
t^{2} \\
t^{3} \\
-t^{1} t^{2} t^{3} \\
t^{2} t^{3} \\
t^{1} t^{3} \\
t^{1} t^{2}
\end{array}\right),
$$

with $\mathcal{R} \subset \operatorname{Sp}(8, \mathbb{Z})$. 
It turns out that $\Pi_{a=1}^{3} \mathrm{SL}(2, \mathbb{Z})_{a}$ and $S_{4}$ symmetries are indeed subgroups of $\operatorname{Sp}(8, \mathbb{Z})$. For instance, the generators of $\mathrm{SL}(2, \mathbb{Z})_{1}$ and $S_{4}$ are chosen as ${ }^{12}$

$$
\begin{aligned}
& S_{1}=\left(\begin{array}{cccccccc}
0 & -1 & 0 & 0 & 0 & 0 & 0 & 0 \\
1 & 0 & 0 & 0 & 0 & 0 & 0 & 0 \\
0 & 0 & 0 & 0 & 0 & 0 & 0 & -1 \\
0 & 0 & 0 & 0 & 0 & 0 & -1 & 0 \\
0 & 0 & 0 & 0 & 0 & -1 & 0 & 0 \\
0 & 0 & 0 & 0 & 1 & 0 & 0 & 0 \\
0 & 0 & 0 & 1 & 0 & 0 & 0 & 0 \\
0 & 0 & 1 & 0 & 0 & 0 & 0 & 0
\end{array}\right), \\
& T_{1}=\left(\begin{array}{llllllll}
1 & 0 & 0 & 0 & 0 & 0 & 0 & 0 \\
1 & 1 & 0 & 0 & 0 & 0 & 0 & 0 \\
0 & 0 & 1 & 0 & 0 & 0 & 0 & 0 \\
0 & 0 & 0 & 1 & 0 & 0 & 0 & 0 \\
0 & 0 & 0 & 0 & 1 & -1 & 0 & 0 \\
0 & 0 & 0 & 0 & 0 & 1 & 0 & 0 \\
0 & 0 & 0 & 1 & 0 & 0 & 1 & 0 \\
0 & 0 & 1 & 0 & 0 & 0 & 0 & 1
\end{array}\right), \\
& P=\left(\begin{array}{llllllll}
1 & 0 & 0 & 0 & 0 & 0 & 0 & 0 \\
0 & 0 & 0 & 1 & 0 & 0 & 0 & 0 \\
0 & 1 & 0 & 0 & 0 & 0 & 0 & 0 \\
0 & 0 & 1 & 0 & 0 & 0 & 0 & 0 \\
0 & 0 & 0 & 0 & 1 & 0 & 0 & 0 \\
0 & 0 & 0 & 0 & 0 & 0 & 0 & 1 \\
0 & 0 & 0 & 0 & 0 & 1 & 0 & 0 \\
0 & 0 & 0 & 0 & 0 & 0 & 1 & 0
\end{array}\right), \\
& Q=\left(\begin{array}{cccccccc}
1 & 0 & 0 & 0 & 0 & 0 & 0 & 0 \\
0 & -1 & 0 & 0 & 0 & 0 & 0 & 0 \\
0 & 0 & 0 & -1 & 0 & 0 & 0 & 0 \\
0 & 0 & 1 & 0 & 0 & 0 & 0 & 0 \\
0 & 0 & 0 & 0 & 1 & 0 & 0 & 0 \\
0 & 0 & 0 & 0 & 0 & -1 & 0 & 0 \\
0 & 0 & 0 & 0 & 0 & 0 & 0 & -1 \\
0 & 0 & 0 & 0 & 0 & 0 & 1 & 0
\end{array}\right),
\end{aligned}
$$

where the fundamental representation of $\operatorname{Sp}(8, \mathbb{Z})$ is split into

$$
8=1+3+1+3
$$

under $S_{4}$ symmetry. We have focused on the transformation of moduli fields, but the $S_{4}$ flavor symmetry of matter fields is also realized by $P$ and $Q$. Indeed, the submatrices of $P$ and $Q$ acting on $\left(t^{1}, t^{2}, t^{3}\right)^{T}$

$$
\left(\begin{array}{lll}
0 & 0 & 1 \\
1 & 0 & 0 \\
0 & 1 & 0
\end{array}\right), \quad\left(\begin{array}{ccc}
-1 & 0 & 0 \\
0 & 0 & -1 \\
0 & 1 & 0
\end{array}\right),
$$

correspond to the transformations for untwisted matters $\left\{A^{1}, A^{2}, A^{3}\right\}$ with the $S_{4}$ triplet. Note that the holomorphic Yukawa coupling is invariant under this $S_{4}$ symmetry.

Let us discuss the origin of the $S_{4}$ symmetry in the effective action. We recall that imaginary parts of moduli $\operatorname{Im}\left(t^{a}\right)$ behave as a triplet representation under $\mathrm{SO}(3)$ group due to the fact that the volume form of factorizable toroidal background $\mathcal{V}$ is given by $\mathcal{V} \simeq \otimes_{a=1}^{3} \operatorname{Im}\left(t^{a}\right) d x^{a} \wedge d y^{a}$. Since the $\mathrm{SO}(3)$ triplet representation corresponds to the $S_{4}$ triplet representation, ${ }^{13} S_{4}$ symmetry exists in the moduli sector $\left\{\operatorname{Im}\left(t^{1}\right), \operatorname{Im}\left(t^{2}\right), \operatorname{Im}\left(t^{3}\right)\right\}$. On the complexified Kähler form, the Kähler moduli consist of volume moduli $\operatorname{Im}\left(t^{a}\right)$ and the Kähler axions $\operatorname{Re}\left(t^{a}\right)$. This fact enhances $\mathrm{SO}(3)$ into $\mathrm{SU}(3)$ symmetry. On the other hand, matter fields are originated from 10D gauge bosons and gauginos. Since 10D $\mathcal{N}=1$

\footnotetext{
${ }^{12}$ It is straightforward to obtain the generators of other $\operatorname{SL}(2, \mathbb{Z})$ by flipping the corresponding moduli fields.

${ }^{13}$ See, e.g., ref. [50] and references therein.
} 
supersymmetry gives rise to $4 \mathrm{D} \mathcal{N}=4$ supersymmetry equipping $\mathrm{SU}(4)_{R}$ symmetry on $T^{6}$ background without orbifolding, the fundamental representation of SU(4) $R$ symmetry can be split into

$$
4=1+3,
$$

under the $\mathrm{SU}(3)_{R}$ symmetry. The matter fields $\left\{A^{a}\right\}$ of our interest have triplet representation of $\mathrm{SU}(3)_{R}$ symmetry, corresponding to the $S_{4}$ triplet representation. Indeed, the $\mathrm{SU}(3)_{R}$ symmetry is embedded into $\operatorname{Sp}(8, \mathbb{C})$ as follows:

$$
R=\left(\begin{array}{cccccccc}
1 & 0 & 0 & 0 & 0 & 0 & 0 & 0 \\
0 & a_{1} & a_{2} & a_{3} & 0 & 0 & 0 & 0 \\
0 & a_{4} & a_{5} & a_{6} & 0 & 0 & 0 & 0 \\
0 & a_{7} & a_{8} & a_{9} & 0 & 0 & 0 & 0 \\
0 & 0 & 0 & 0 & 1 & 0 & 0 & 0 \\
0 & 0 & 0 & 0 & 0 & a_{1} & a_{2} & a_{3} \\
0 & 0 & 0 & 0 & 0 & a_{4} & a_{5} & a_{6} \\
0 & 0 & 0 & 0 & 0 & a_{7} & a_{8} & a_{9}
\end{array}\right), \quad \text { with }\left(\begin{array}{lll}
a_{1} & a_{2} & a_{3} \\
a_{4} & a_{5} & a_{6} \\
a_{7} & a_{8} & a_{9}
\end{array}\right) \in \mathrm{SU}(3)_{R}
$$

where we used that $t^{1} t^{2} t^{3}\left(A^{1} A^{2} A^{3}\right)$ is a $\mathrm{SU}(3)_{R}$ singlet.

In this way, $S_{4}$ flavor symmetry naturally lies in the symplectic modular flavor symmetry. Together with the CP transformation as discussed in section 2.4, the symplectic modular symmetry is generalized to the generalized symplectic modular symmetry. It turns out that the $S_{4}$ flavor symmetry is enhanced to

$$
S_{4} \rtimes \mathbb{Z}_{2}^{\mathrm{CP}},
$$

in a generic moduli space of $\left\{t^{a}\right\}$. Here, $\mathbb{Z}_{2}^{\mathrm{CP}}$ transformation is given by eqs. (2.49) and (2.50).

\subsection{Small resolutions of $T^{6} / \mathbb{Z}_{3}$ orbifold}

In this section, we incorporate the twisted (blow-up) modes in the effective action on the $6 \mathrm{D}$ toroidal orbifolds. We mainly deal with the blown-up $T^{6} / \mathbb{Z}_{3}$ orbifold, which is directly connected with the effective action of untwisted modes in the previous section in the blow-down limit, i.e., the vanishing blow-up radii. On the blown-up $T^{6} / \mathbb{Z}_{3}$ geometry, only the Kähler structure deformations remain on the blown-up $T^{6} / \mathbb{Z}_{3}$ background. The totally $h^{1,1}=36$ Kähler moduli include $h_{\text {untw }}^{1,1}=9$ untwisted Kähler moduli and $h_{\mathrm{tw}}^{1,1}=27$ twisted Kähler moduli $c^{d}(d=1,2, \cdots, 27)$ from the viewpoint of toroidal orbifold. ${ }^{14}$ For simplicity, let us focus on the three diagonal untwisted Kähler moduli $t^{a}(a=1,2,3)$ whose moduli space is given by $\Pi_{a} \mathrm{SL}(2, \mathbb{Z})_{a}$. The prepotential receives the corrections from the blow-up modes [36]

$$
G=t^{1} t^{2} t^{3}-\frac{i}{4} \sum_{d=1}^{27}\left(c^{d}\right)^{2},
$$

\footnotetext{
${ }^{14}$ The following discussion is applicable to the T-dual of $T^{6} / \mathbb{Z}_{3}$ orbifold, where the massless deformations correspond to the complex structure moduli accompanying the $\overline{27}_{i}$ matter fields. For more details, see, ref. [51] and references therein.
} 
and the following vector

$$
\left(\begin{array}{c}
Y^{A} \\
\mathcal{G}_{A}
\end{array}\right)=\left(\begin{array}{c}
1 \\
t^{1} \\
t^{2} \\
t^{3} \\
c^{d} \\
2 G-t^{A} \partial_{A} G \\
t^{2} t^{3} \\
t^{1} t^{3} \\
t^{1} t^{2} \\
-\frac{i}{2} c^{d}
\end{array}\right)=\left(\begin{array}{c}
1 \\
t^{1} \\
t^{2} \\
t^{3} \\
c^{d} \\
-t^{1} t^{2} t^{3} \\
t^{2} t^{3} \\
t^{1} t^{3} \\
t^{1} t^{2} \\
-\frac{i}{2} c^{d}
\end{array}\right)
$$

provides the Kähler potential

$$
K_{\mathrm{ks}}=-\ln \left[-i\left(\bar{Y}^{A} \mathcal{G}_{A}-Y^{A} \overline{\mathcal{G}}_{A}\right)\right]=-\ln \left[i\left(t^{1}-\bar{t}^{1}\right)\left(t^{2}-\bar{t}^{2}\right)\left(t^{3}-\bar{t}^{3}\right)-\sum_{d}\left|c^{d}\right|^{2}\right]
$$

which is consistent with ref. [16]. Correspondingly, the matter Kähler metric is calculated through eq. (2.24).

The allowed Yukawa couplings of matter zero-modes are restricted by $\mathbb{Z}_{3}$ symmetry. The matter superpotential takes the form

$$
W=A^{1} A^{2} A^{3}-\sum_{d, e, f=1}^{27} f\left(t^{1}, t^{2}, t^{3}\right)_{d e f} C^{d} C^{e} C^{f}
$$

where $C^{d}$ denotes the twisted matters associated twisted Kähler moduli, and their Yukawa couplings $f\left(t^{1}, t^{2}, t^{3}\right)$ def are moduli-dependent in general [52-54]. The holomorphic Yukawa couplings of untwisted modes enjoy the $S_{4}$ flavor symmetry as previously discussed in the orbifold regime, but the flavor symmetry of the twisted matter fields is complicated. Before going into the detail of the Yukawa couplings among twisted matters, we comment on the $S_{4}$ symmetry of twisted matter fields. Let us denote them by fixed point labels, $C_{n_{1}, n_{2}, n_{3}}$, where $n_{i}=0,1,2$ represents the fixed point label on the $i$-th $T^{2} / \mathbb{Z}_{3}$. The matter fields $C_{n, n, n}$ are obviously $S_{4}$ singlets. The other $C_{n_{1}, n_{2}, n_{3}}$ are triplets because they transform each other under $S_{4}$. Thus, 27 twisted matter fields decompose into three $S_{4}$ singlets and eight $S_{4}$ triplets.

In the blow-down limit treated in this section, the relevant modular symmetry in the untwisted Kähler moduli space is $\Pi_{a=1}^{3} \operatorname{SL}(2, \mathbb{Z})_{a} \subset \operatorname{Sp}(8, \mathbb{Z})$. It was known that the Kähler metrics of untwisted and twisted matters take the form $[18,36]$

$$
\begin{aligned}
\left.K_{a \bar{b}}^{(27)}\right|_{\mathrm{untw}} & =\left(K_{\mathrm{ks}}\right)_{a \bar{b}} e^{-K_{a}}=\frac{1}{i\left(t^{a}-\bar{t}^{a}\right)} \delta_{a \bar{b}}, \\
\left.K_{d \bar{e}}^{(27)}\right|_{\mathrm{tw}} & =\left(K_{\mathrm{ks}}\right)_{d \bar{e}} e^{-K_{\mathrm{ks}} / 3}=\frac{1}{\left\{i\left(t^{1}-\bar{t}^{1}\right)\left(t^{2}-\bar{t}^{2}\right)\left(t^{3}-\bar{t}^{3}\right)\right\}^{2 / 3}} \delta_{d \bar{e}},
\end{aligned}
$$


with $K_{a}=-\ln \left(-i\left(t^{a}-\bar{t}^{a}\right)\right)$, from which we deduce the modular weight of both matter fields:

$$
A^{1}:\{-1,0,0\}, \quad A^{2}:\{0,-1,0\}, \quad A^{3}:\{0,0,-1\}, \quad C^{d}:\left\{-\frac{2}{3},-\frac{2}{3},-\frac{2}{3}\right\},
$$

under $\left\{\mathrm{SL}(2, \mathbb{Z})_{1}, \mathrm{SL}(2, \mathbb{Z})_{2}, \mathrm{SL}(2, \mathbb{Z})_{3}\right\}$. These modular weights play an important role in checking the modular invariance of the action. Indeed, the matter superpotential of untwisted modes $A^{1} A^{2} A^{3}$ is invariant under the $\Pi_{a} \mathrm{SL}(2, \mathbb{Z})_{a}$ modular symmetry due to its modular weight -1 under all $\operatorname{SL}(2, \mathbb{Z})_{a}$. However, 3-point couplings of twisted modes $C^{d} C^{e} C^{f}$ have modular weight -2 under all $\operatorname{SL}(2, \mathbb{Z})_{a}$. Hence, the modular invariance requires the moduli-dependent couplings $f\left(t^{1}, t^{2}, t^{3}\right)$ def with modular weight 1 for each $\mathrm{SL}(2, \mathbb{Z})_{a}$. It was known that the twisted modes belong to the triplet representation of double cover of $\left(A_{4}\right)_{a}$, namely $\left(T^{\prime}\right)_{a} \subset \mathrm{SL}(2, \mathbb{Z})_{a}$ in the blow-down limit $[16,55]$, although this $T^{\prime}$ triplet is a reducible representation as seen below. Their holomorphic Yukawa couplings are expected to be a $\Gamma(3)$ modular form of non-Abelian discrete group $A_{4}$, which bring into the $\left(T^{\prime}\right)_{a}$-invariant superpotential. We will come back to this point later.

To see the $T^{\prime}$ structure in more detail, let us discuss $T^{\prime}$ transformations in the symplectic basis. Note that on this blown-up geometry, $S_{4}$ symmetry among untwisted modes lies in $\operatorname{Sp}(8, \mathbb{Z}) \subset \mathrm{Sp}(62, \mathbb{Z})$ in a way similar to the previous analysis. For concreteness, we focus on a subsector of twisted modes, i.e., three twisted fields $\left\{C^{1}, C^{2}, C^{3}\right\}$ which transform under the $\mathrm{SL}(2, \mathbb{Z})_{1}$ symmetry. Then, the representations of these twisted matters under the $S_{1}$ and $T_{1}$ transformations of $\mathrm{SL}(2, \mathbb{Z})_{1}$ are given by $[16,55]$

$$
\mathcal{S}_{\mathrm{tw}}=-\frac{i}{\sqrt{3}}\left(\begin{array}{ccc}
1 & 1 & 1 \\
1 & w & w^{2} \\
1 & w^{2} & w
\end{array}\right), \quad \mathcal{T}_{\mathrm{tw}}=\left(\begin{array}{ccc}
w & 0 & 0 \\
0 & 1 & 0 \\
0 & 0 & 1
\end{array}\right)
$$

with $w=e^{2 \pi i / 3}$. They satisfy the following algebraic relations:

$$
\mathcal{S}_{\mathrm{tw}}^{2}=-\mathbb{I}, \quad\left(\mathcal{S}_{\mathrm{tw}} \mathcal{T}_{\mathrm{tw}}\right)^{3}=\mathcal{T}_{\mathrm{tw}}^{3}=\mathbb{I},
$$

which is the $T^{\prime}$ symmetry $[47,48]$. Note that these matrices are rotated into

$$
\mathcal{U}_{\mathrm{tw}}^{-1} \mathcal{S}_{\mathrm{tw}} \mathcal{U}_{\mathrm{tw}}=-\frac{i}{\sqrt{3}}\left(\begin{array}{ccc}
1 & \sqrt{2} & 0 \\
\sqrt{2} & -1 & 0 \\
0 & 0 & i
\end{array}\right), \quad \mathcal{U}_{\mathrm{tw}}^{-1} \mathcal{T}_{\mathrm{tw}} \mathcal{U}_{\mathrm{tw}}=\mathcal{T}_{\mathrm{tw}}
$$

by the unitary transformation:

$$
\mathcal{U}_{\mathrm{tw}}=\left(\begin{array}{ccc}
1 & 0 & 0 \\
0 & \frac{1}{\sqrt{2}} & -\frac{1}{\sqrt{2}} \\
0 & \frac{1}{\sqrt{2}} & \frac{1}{\sqrt{2}}
\end{array}\right)
$$

It indicates that $T^{\prime}$ triplet of our interest is a reducible representation decomposed into the singlet and the doublet representations. 
We find that these transformations lie in the symplectic modular group as follows:

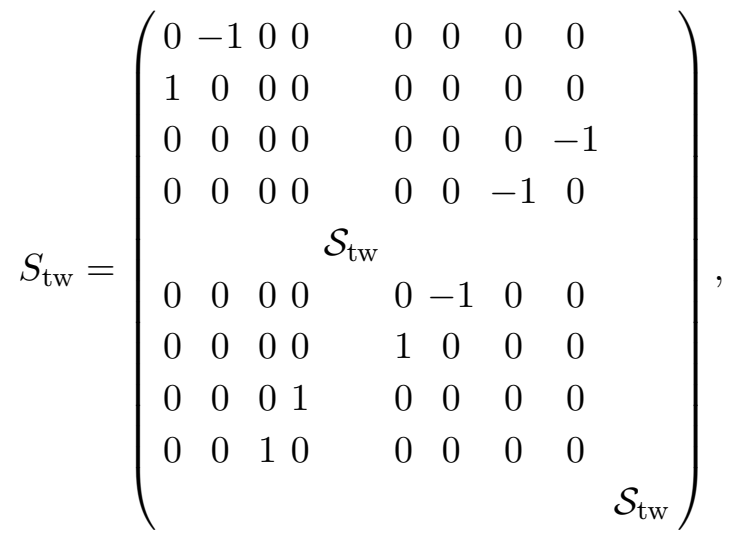

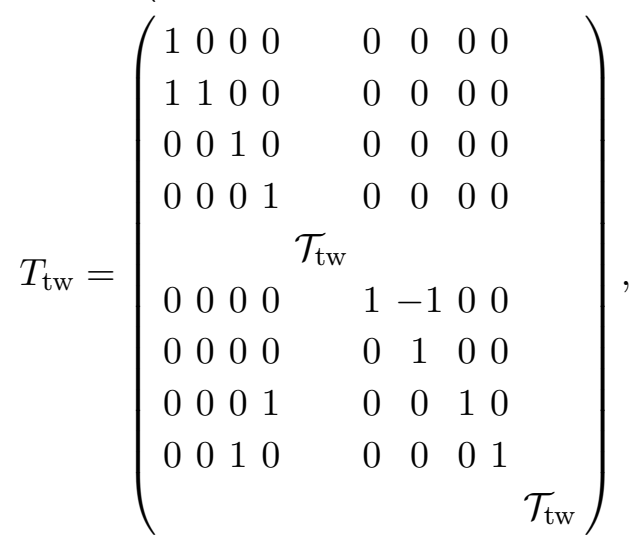

where we omit 0 in the blank space for simplicity. Here, we focused on three twisted modes, but it is straightforward to extend this analysis on fully resolved toroidal orbifolds with small resolutions. In that case, the effective action enjoys the $\left(T^{\prime}\right)^{3}$ flavor symmetry belonging to $\operatorname{Sp}(62, \mathbb{Z})$ modular symmetry.

Similar to the untwisted modes, $T^{\prime}$ flavor symmetry naturally lies in the symplectic modular flavor symmetry because it is a quotient of $\mathrm{SL}(2, \mathbb{Z})$. The twisted modes have non-trivial representations under

$$
\left(T^{\prime}\right)^{3} \times S_{4}
$$

Thus, their vacuum expectation values may lead non-trivial breaking of $\left(T^{\prime}\right)^{3} \times S_{4}$. Together with the CP transformation as discussed in section 2.4, symplectic modular symmetry is enlarged to the generalized symplectic modular symmetry. In the current setup, each $T^{\prime}$ flavor symmetry is enhanced to

$$
T^{\prime} \rtimes \mathbb{Z}_{2}^{\mathrm{CP}} \simeq S_{4},
$$

as an element of $G \operatorname{Sp}(62, \mathbb{Z})$. Note that $\mathbb{Z}_{2}^{\mathrm{CP}}$ transformation exchanges between the irreducible representations of $T^{\prime}$, i.e., the outer automorphism of $T^{\prime}$.

To understand the nature of Yukawa couplings among twisted matters, we remind the couplings of twisted matters on $T^{2} / \mathbb{Z}_{3}$ geometry whose fixed points are labeled by $n=0,1,2$. The total 27 fixed points on $T^{6} / \mathbb{Z}_{3}$ orbifold is reproduced by the product 
of three fixed points on $T^{2} / \mathbb{Z}_{3}$. As a consequence of $\mathbb{Z}_{3}$ symmetry, Yukawa couplings of twisted modes localized on

$$
(0,0,0), \quad(1,1,1), \quad(2,2,2), \quad(0,1,2)
$$

are only allowed on $T^{2} / \mathbb{Z}_{3}$. In particular, their Yukawa couplings are of the form [52-54]

$$
f\left(t^{1}, t^{2}, t^{3}\right)_{\operatorname{def}} \propto e^{-\mathcal{A}}
$$

where $\mathcal{A}$ denotes the area satisfying boundary conditions relevant to twisted strings. Furthermore, these twisted strings wrap the cycle of toroidal orbifolds many times. Summing up these effects, the Yukawa couplings

$$
f\left(t^{1}, t^{2}, t^{3}\right)_{\operatorname{def}} \propto \sum_{m} e^{-\mathcal{A}_{m}}
$$

will be elliptic functions such as theta functions as explicitly demonstrated on orbifolds. Note that the leading terms of holomorphic Yukawa couplings for $(n, n, n)$ with $n=0,1,2$ are constant, but the other one $(0,1,2)$ is exponentially suppressed with respect to the torus radii due to the fact that twisted strings propagate the fixed points with another. That is world-sheet instanton effects. The couplings among the fixed points $(0,1,2)$ would vanish if we neglect such world-sheet instanton effects.

Let us revisit these results in the context of symplectic modular symmetry, focusing on the modular transformation of Yukawa couplings on $T^{2} / \mathbb{Z}_{3}$. We denote the Yukawa coupling among three twisted modes on the same fixed point by $Y_{0}$, while we denote the Yukawa couplings for three different fixed points $(0,1,2)$ and $(2,1,0)$ by $Y_{1}$ and $Y_{2}$, following refs. [56, 57]. The triplet $\left(Y_{0}, Y_{1}, Y_{2}\right)$ transforms in the same way as the three twisted modes, i.e., eq. (3.20), up to an overall phase. Thus, they decompose the singlet and doublet under $T^{\prime}$, but the singlet corresponds to $\left(Y_{1}-Y_{2}\right)$ and it vanishes automatically. The doublet corresponds to the modular forms of weight 1 for $T^{\prime}$. Indeed, the modular forms of weight 1 for $T^{\prime}$ have two degrees of freedom, corresponding to the $T^{\prime}$ doublet [58]. Appendix A shows the modular form of weight 1 for $T^{\prime}$ in a representation basis. ${ }^{15}$ Indeed, this fact is well in accord with our observations. The constant Yukawa couplings localized at the fixed points $(n, n, n)$ with $n=0,1,2$ are the remnant of such modular functions in the large volume regime, whereas other one at $(0,1,2)$ are exponentially suppressed due to the structure of elliptic functions. The large volume expansion is nothing but the $q=e^{2 \pi i t}$ expansion in the modular form. So far, we have focused on $T^{2} / \mathbb{Z}_{3}$ orbifold, but the structure discussed above holds for the Yukawa couplings of twisted modes associated with 27 fixed points on $T^{6} / \mathbb{Z}_{3}$ orbifold.

In the limit that the values of blow-up moduli are sufficiently large, 27 twisted modes may have the flavor symmetry $S_{27}$ in $\operatorname{Sp}(54, \mathbb{Z})$ modular symmetry as the model in section 3.4. However, in the blow-down orbifold limit, the symmetry $S_{27}$ as well as $\operatorname{Sp}(54, \mathbb{Z})$ loses the geometrical meaning. However, twisted modes transform non-trivially under the

\footnotetext{
${ }^{15}$ Similar to twisted modes and their Yukawa couplings, zero-modes and their Yukawa couplings in magnetized D-brane models have non-trivial behavior under the modular symmetry [59-66].
} 
$\mathrm{SL}(2, \mathbb{Z})$ modular group of untwisted modes as the $T^{\prime}$ reducible triplet because of the structure of prepotential (3.14) and Kähler potential (3.16). That is a quite trivial transition of modular symmetric behavior of the twisted modes. However, we have understood just two regimes, i.e., sufficiently large blow-up moduli regime and the blow-down orbifold limit. The middle regime is unclear in our analysis. We hope to report on the whole picture of modular symmetric behavior in the future.

Finally, we comment on the other untwisted moduli whose moduli space is given by $\frac{\mathrm{SU}(3,3)}{\mathrm{SU}(3) \times \mathrm{SU}(3) \times \mathrm{U}(1)}$. From the prepotential of the untwisted modes

$$
G=\sum_{a, b, c=1}^{9} \kappa_{a b c} t^{a} t^{b} t^{c}
$$

with

$$
\kappa_{123}=\kappa_{468}=\kappa_{579}=1, \quad \kappa_{169}=\kappa_{258}=\kappa_{347}=-1,
$$

and 0 otherwise, the Kähler potential of untwisted modes is provided by

$$
K_{\mathrm{ks}}=-\ln \left[-i \frac{\kappa_{a b c}}{6}\left(t^{a}-\bar{t}^{a}\right)\left(t^{b}-\bar{t}^{b}\right)\left(t^{c}-\bar{t}^{c}\right)\right] .
$$

Here, we take the $Y^{0}=1$ gauge. It is also known that the matter Käher potential and corresponding metric are given by [67]

$$
\begin{aligned}
K & =-\ln \operatorname{det}\left(-i(\mathcal{T}-\overline{\mathcal{T}})-A_{\alpha} A_{\bar{\alpha}}\right)^{\hat{a} \hat{b}}=K_{\mathrm{ks}}+(-i(\mathcal{T}-\overline{\mathcal{T}}))_{\hat{a} \hat{b}}^{-1} A_{\alpha}^{\hat{a}} A_{\bar{\alpha}}^{\overline{\hat{b}}}+\mathcal{O}\left(|A|^{4}\right), \\
K_{\hat{a} \hat{\hat{b}}}^{(27)} & =(-i(\mathcal{T}-\overline{\mathcal{T}}))_{\hat{a} \hat{\hat{b}}}^{-1},
\end{aligned}
$$

where

$$
\mathcal{T}=\left(\begin{array}{lll}
t^{1} & t^{4} & t^{5} \\
t^{7} & t^{2} & t^{6} \\
t^{8} & t^{9} & t^{3}
\end{array}\right),
$$

with $\hat{a}, \hat{b}=1,2,3$. Note that $\alpha$ stands for the indices of $\mathrm{SU}(3) \subset E_{8}$ gauge group which are different from the indices of $\mathrm{SU}(3)$ isometry $\hat{a}$. The superpotential of untwisted modes is written by ${ }^{16}$

$$
W=\sum_{\hat{a}, \hat{b}, \hat{c}, \alpha, \beta, \gamma} \kappa_{\hat{a} \hat{b} \hat{c}} \epsilon^{\alpha \beta \gamma} A_{\alpha}^{\hat{a}} A_{\beta}^{\hat{b}} A_{\gamma}^{\hat{c}},
$$

with $\kappa_{\hat{1} \hat{2} \hat{3}}=1$ and 0 otherwise, up to the overall factor, where $\epsilon^{\alpha \beta \gamma}$ are the anti-symmetric tensors of $\mathrm{SU}(3) \subset E_{8}$ gauge group, and the upper and lower indices correspond to the $\mathrm{SU}(3)$ isometry and $\mathrm{SU}(3) \subset E_{8}$ gauge group, respectively. The $S_{4}$ flavor symmetry appears in three untwisted matters, e.g., $\left\{A_{1}^{\hat{1}}, A_{1}^{\hat{2}}, A_{1}^{\hat{3}}\right\}$ which can be embedded into the symplectic modular group following the previous procedures. In this way, the 9 untwisted modes transform as 3 triplet representations under the $S_{4}$ symmetry, that is, $\left\{A_{\alpha}^{\hat{a}}, A_{\alpha}^{\hat{b}}, A_{\alpha}^{\hat{c}}\right\}$ for arbitrary $\alpha$.

\footnotetext{
${ }^{16}$ The kinetic mixing of matter fields will play an important role of realizing the hierarchical structure of matter fields [68].
} 


\subsection{Small resolutions of $T^{6} /\left(\mathbb{Z}_{3} \times \mathbb{Z}_{3}^{\prime}\right)$ orbifold}

We deal with another example to exemplify the existence of generalized symplectic modular symmetry. We focus on the small resolutions of $T^{6} /\left(\mathbb{Z}_{3} \times \mathbb{Z}_{3}^{\prime}\right)$ orbifold by further imposing $\mathbb{Z}_{3}^{\prime}$ projection on $T^{6} / \mathbb{Z}_{3}$ orbifold. By introducing the complex coordinates of $\mathbb{C}^{3}$ as $z^{m}$, $m=1,2,3$, we identify them with

$$
z^{m} \simeq z^{m}+1, \quad z^{m} \simeq z^{m}+\beta,
$$

with $\beta=e^{2 \pi i / 6}$ to construct the three torus. Then, we specify the $\mathbb{Z}_{3} \times \mathbb{Z}_{3}^{\prime}$ action following ref. [43]:

$$
\begin{aligned}
& \mathbb{Z}_{3}: Q\left(z^{m}\right) \simeq \beta^{2} z^{m}, \\
& \mathbb{Z}_{3}^{\prime}: Q^{\prime}\left(z^{m}\right) \simeq \beta^{2 m} z^{m}+\frac{1+\beta}{3},
\end{aligned}
$$

where $\mathbb{Z}_{3}^{\prime}$ is a freely acting symmetry. From 27 fixed points under $\mathbb{Z}_{3}$ symmetry

$$
z_{\text {fix }}=\frac{1+\beta}{3}\left(n_{1}, n_{2}, n_{3}\right),
$$

with $n_{m}=0,1,2$, we find 9 orbifold-invariant combinations:

$$
\begin{array}{ll}
f_{1}:(0,0,0)+(1,1,1)+(2,2,2), & f_{2}:(0,1,2)+(1,2,0)+(2,0,1), \\
f_{3}:(0,2,1)+(1,0,2)+(2,1,0), & \\
f_{4}:(1,0,0)+(2,1,1)+(0,2,2), & f_{5}:(0,1,0)+(1,2,1)+(2,0,2), \\
f_{6}:(0,0,1)+(1,1,2)+(2,2,0), & \\
f_{7}:(2,0,0)+(0,1,1)+(1,2,2), & f_{8}:(0,2,0)+(1,0,1)+(2,1,2), \\
f_{9}:(0,0,2)+(1,1,0)+(2,2,1) . &
\end{array}
$$

Hence, there exist $h_{\mathrm{tw}}^{1,1}=9$ twisted Kähler moduli in addition to $h_{\mathrm{untw}}^{1,1}=3$ untwisted Kähler moduli.

The orbifold projection (3.37) breaks $\left(T^{\prime}\right)^{3}$ symmetry in section 3.2 to $S_{3}$. For instance, eq. (3.39) represents the $S_{3}$ singlet, and eqs. (3.40) and (3.41) become $S_{3}$ triplets, although the triplet representation is the reducible representation decomposed into the single and doublet. In addition, 27 twisted modes on $T^{6} / \mathbb{Z}_{3}$ correspond to three singlets and eight triplets under the $S_{4}$ flavor symmetry of untwisted modes, where the matter fields $C_{n, n, n}$ correspond to the $S_{4}$ singlets, while the other $C_{n_{1}, n_{2}, n_{3}}$ are $S_{4}$ triplets. All of the matter fields $C_{n, n, n}$ correspond to three fixed points in the linear combination $f_{1}$. Here, the matter fields corresponding to both $f_{2}$ and $f_{3}$ become $S_{4}$ singlets because of the orbifold projection (3.37). On the other hand, the matter fields corresponding to $\left(f_{4}, f_{5}, f_{6}\right)$ and $\left(f_{7}, f_{8}, f_{9}\right)$ still represent the $S_{4}$ triplets. Thus, the orbifold projection (3.37) reduces three $S_{4}$ singlets and eight $S_{4}$ triplets to three $S_{4}$ singlets and two $S_{4}$ triplets. From the total $\operatorname{Sp}(2 \times 12+2, \mathbb{Z})$ symplectic group, we can derive the $S_{3}$ flavor group,

$$
\operatorname{Sp}(26, \mathbb{Z}) \supset G_{\text {flavor }}=S_{3},
$$


where the embedding $S_{3} \subset \operatorname{Sp}(8, \mathbb{Z}) \subset \operatorname{Sp}(26, \mathbb{Z})$ is realized by following the same step in the previous section. The $S_{3}$ generator corresponds to $P$ in eq. (3.5). Then, the $S_{3}$ flavor symmetry is enlarged to $D_{6} \simeq S_{3} \rtimes \mathbb{Z}_{2}^{\mathrm{CP}} \subset G \mathrm{Sp}(26, \mathbb{Z})$, taking into account the CP symmetry. Here, $\mathbb{Z}_{2}^{C P}$ transformation is given by eqs. (2.49) and (2.50).

In the following, we move to the geometrical regime where the size of blow-up radii is above the string length and check whether there exist such discrete modular flavor symmetries.

\subsection{Geometrical regime of blown-up $T^{6} /\left(\mathbb{Z}_{3} \times \mathbb{Z}_{3}^{\prime}\right)$ orbifold}

In the previous sections, we examined the blown-up toroidal orbifolds with small resolutions, where the $S_{3}, S_{4}$, and $T^{\prime}$ flavor symmetries among untwisted and twisted matters are independent. Together with the CP symmetry, all of them belong to the generalized symplectic modular symmetry and are enlarged to $D_{6}, S_{4} \rtimes \mathbb{Z}_{2}^{\mathrm{CP}}$ and $S_{4}$, respectively.

In contrast to the previous analysis assuming the small vacuum expectation values of blow-up modes, we discuss more non-trivial examples on smooth CY threefolds. In this section, we focus on $T^{6} /\left(\mathbb{Z}_{3} \times \mathbb{Z}_{3}^{\prime}\right)$ background, where the blown-up cycles are larger than 1 in string units, in comparison with the analysis of section 3.3. Among $h^{1,1}=12$ Kähler moduli, there exist $h_{\text {untw }}^{1,1}=3$ untwisted Kähler moduli spanned by $e_{a}$ corresponding to $i d z^{a} \wedge d \bar{z}^{\bar{b}}$ from the viewpoint of toroidal orbifold, where $z^{a}(a=1,2,3)$ denote the coordinates of 3-torus. In addition, we have $h_{\mathrm{tw}}^{1,1}=9$ twisted Kähler moduli spanned by $e_{r}$ $(r=1,2, \ldots, 9)$, corresponding to complex projective spaces $\mathbb{P}^{2}$ 's, where fixed points are now resolved as $\mathbb{P}^{2}$ of a $\mathbb{C}^{3} / \mathbb{Z}_{3}$ singularity. On this geometry, there is no complex structure deformation.

When we expand the Kähler form

$$
J=\sum_{a=1}^{3} t^{a} e_{a}+\sum_{r=1}^{9} v^{r} e_{r},
$$

the Kähler potential is evaluated as [69]

$$
K_{\mathrm{ks}}=-\ln \int J_{c} \wedge J_{c} \wedge J_{c}=-\ln \frac{1}{8}\left[81\left(t^{1}-\bar{t}^{1}\right)\left(t^{2}-\bar{t}^{2}\right)\left(t^{3}-\bar{t}^{3}\right)-\frac{3}{2} \sum_{r}\left(v^{r}-\bar{v}^{r}\right)^{3}\right] .
$$

Here, the prepotential is given by

$$
G=\frac{\kappa_{A B C}}{6} t^{A} t^{B} t^{C}=81 t^{1} t^{2} t^{3}-\frac{3}{2} \sum_{r}\left(v^{r}\right)^{3}
$$

with the nonvanishing triple intersection numbers,

$$
\kappa_{123}=81, \quad \kappa_{r r r}=-9 .
$$

Hence, the corresponding matter superpotential takes the form

$$
W=81 A^{1} A^{2} A^{3}-9 \sum_{r=1}^{9}\left(V^{r}\right)^{3},
$$


where $A^{1,2,3}$ and $V^{r}$ denote the matter fields associated with the untwisted and twisted moduli fields from the viewpoint of toroidal orbifolds, respectively. The matter Kähler metric is calculated through eq. (2.24). We find that the matter superpotential is invariant under two flavor symmetries: $S_{4}$ symmetry for the untwisted matters $\left\{A^{1}, A^{2}, A^{3}\right\}$ and $S_{9}$ symmetry for the twisted matters $\left\{V^{1}, V^{2}, \ldots, V^{9}\right\}$ in addition to the overall modular symmetry $\operatorname{SL}(2, \mathbb{Z})_{\text {overall }}$.

To see the relation between flavor symmetries of matter fields and symplectic modular symmetry, let us consider the period vector providing the Kähler potential (3.44). Since the blown-up $T^{6} /\left(\mathbb{Z}_{3} \times \mathbb{Z}_{3}^{\prime}\right)$ manifold have totally $12 \mathrm{Kähler} \mathrm{moduli} \mathrm{fields,} \mathrm{the} \mathrm{moduli} \mathrm{space} \mathrm{is}$ described by $\operatorname{Sp}(26, \mathbb{Z})$ symplectic modular symmetry. Correspondingly, the period vector is given by

$$
\left(\begin{array}{c}
Y^{A} \\
\mathcal{G}_{A}
\end{array}\right)=\left(\begin{array}{c}
1 \\
t^{1} \\
t^{2} \\
t^{3} \\
v^{r} \\
2 G-t^{A} \partial_{A} G \\
81 t^{2} t^{3} \\
81 t^{1} t^{3} \\
81 t^{1} t^{2} \\
-\frac{9}{2}\left(v^{r}\right)^{2}
\end{array}\right)=\left(\begin{array}{c}
1 \\
t^{1} \\
t^{2} \\
t^{3} \\
v^{a} \\
-81 t^{1} t^{2} t^{3}+\frac{3}{2} \sum_{r}\left(v^{r}\right)^{3} \\
81 t^{2} t^{3} \\
81 t^{1} t^{3} \\
81 t^{1} t^{2} \\
-\frac{9}{2}\left(v^{r}\right)^{2}
\end{array}\right) .
$$

Similar to the previous example, the $S_{4}$ flavor symmetry of the untwisted moduli $\left\{t^{1}, t^{2}, t^{3}\right\}$ can be embedded into $\operatorname{Sp}(8, \mathbb{Z}) \subset \operatorname{Sp}(26, \mathbb{Z})$. Accordingly, the matter fields $A^{1,2,3}$ transform as in eq. (3.10). In addition, we find that the generators of $S_{9}$

$$
\mathcal{P}_{r s}: v^{r} \leftrightarrow v^{s}
$$

is the element of $\operatorname{Sp}(26, \mathbb{Z})$ as follows. For an illustrative purpose, let us focus on $\mathcal{P}_{12}$ exchanging $v^{1}$ and $v^{2}$. To shorten the expression of the generator $\mathcal{P}_{12}$, we focus on the relevant period vector transformed under $\mathcal{P}_{12}$,

$$
\left(\begin{array}{c}
v^{1} \\
v^{2} \\
-\frac{9}{2}\left(v^{1}\right)^{2} \\
-\frac{9}{2}\left(v^{2}\right)^{2}
\end{array}\right)
$$

on which $\mathcal{P}_{12}$ acts on

$$
\left(\begin{array}{llll}
0 & 1 & 0 & 0 \\
1 & 0 & 0 & 0 \\
0 & 0 & 0 & 1 \\
0 & 0 & 1 & 0
\end{array}\right)\left(\begin{array}{c}
v^{1} \\
v^{2} \\
-\frac{9}{2}\left(v^{1}\right)^{2} \\
-\frac{9}{2}\left(v^{2}\right)^{2}
\end{array}\right)=\left(\begin{array}{c}
v^{2} \\
v^{1} \\
-\frac{9}{2}\left(v^{2}\right)^{2} \\
-\frac{9}{2}\left(v^{1}\right)^{2}
\end{array}\right) .
$$


$\mathcal{P}_{12}$ acts the identity operators on the other elements of period vector. In this way, we show that the generator $\mathcal{P}_{12}$ is the element of $\operatorname{Sp}(26, \mathbb{Z})$, and it is possible to prove the other $\mathcal{P}_{r s}$ as the element of $\operatorname{Sp}(26, \mathbb{Z})$ by changing the indices of $v^{r, s}$.

These transformations are directly related to the $S_{9}$ flavor symmetry of matter fields from $\mathcal{P}_{r s}$. Indeed, the submatrix of $\mathcal{P}_{r s}$ acting on $\left(v^{1}, v^{2}\right)^{T}$

$$
\left(\begin{array}{ll}
0 & 1 \\
1 & 0
\end{array}\right)
$$

corresponds to the transformation between $V^{1}$ and $V^{2}$. Combining all $\mathcal{P}_{a b}$ acting on

$$
\left(v^{1}, v^{2}, v^{3}, v^{4}, v^{5}, v^{6}, v^{7}, v^{8}, v^{9}\right)^{T},
$$

one can realize the transformations of matter fields under $S_{9}$ modular flavor symmetry.

We conclude that there exist $G_{\text {flavor }}=S_{4} \times S_{9}$ flavor symmetries in the effective action of blown-up $T^{6} /\left(\mathbb{Z}_{3} \times \mathbb{Z}_{3}^{\prime}\right)$ orbifold. They originate from the $\operatorname{Sp}(26, \mathbb{Z})$ symplectic group,

$$
\operatorname{Sp}(26, \mathbb{Z}) \supset G_{\text {flavor }}=S_{4} \times S_{9},
$$

where $S_{9}$ symmetry is broken down to $S_{3}$ in the regime with small resolutions as in section 3.3. That would be related to the spontaneous breaking of flavor symmetry triggered by the blow-up modes. We will leave the more detailed discussion of this symmetry breaking involving the stabilization of blow-up modes to future work. When we take into account the CP transformation, two flavor symmetries are enhanced to

$$
S_{4} \rtimes \mathbb{Z}_{2}^{\mathrm{CP}}, \quad S_{9} \rtimes \mathbb{Z}_{2}^{\mathrm{CP}} .
$$

In the next section, we show the existence of non-Abelian discrete flavor symmetries on other classes of CY threefolds.

This example suggests that some class of models with $h$ blow-up modes may lead to the superpotential

$$
W=\sum_{i=1}^{h}\left(A^{i}\right)^{3}
$$

up to an overall factor. This superpotential seems to have the $S_{h}$ flavor symmetry, which is the permutation among $A^{i}$. This type of superpotential has a specific property for $h=3$. We start with the following superpotential:

$$
W=\tilde{A}^{1} \tilde{A}^{2} \tilde{A}^{3} .
$$

Here, we redefine the matter fields,

$$
\begin{aligned}
& \tilde{A}^{1}=A^{1}+A^{2}+A^{3}, \\
& \tilde{A}^{1}=A^{1}+\omega A^{2}+\omega^{2} A^{3}, \\
& \tilde{A}^{1}=A^{1}+\omega^{2} A^{2}+\omega A^{3} .
\end{aligned}
$$

Then, we can write

$$
W=\tilde{A}^{1} \tilde{A}^{2} \tilde{A}^{3}=\left(A^{1}\right)^{3}+\left(A^{2}\right)^{2}+\left(A^{3}\right)^{2} .
$$

The $S_{3}$ symmetry is enlarged to $S_{4}$. Hence, this phenomenon can be observed only for $h=3$. 


\subsection{Three-parameter examples of Calabi-Yau threefolds}

So far, we have focused on the toroidal orbifolds with and without resolutions. In this section, we analyze the smooth CY threefolds, emphasizing the flavor symmetry of matter fields. In particular, we deal with the Kreuzer-Skarke data set of CY threefolds, given by total 473,800,776 ambient toric fourfolds [70]. The ambient toric varieties are described by the corresponding (reflexive) polytope, which might involve singularities in general. The smooth CY hypersurfaces are obtained through "triangulation" of the polytope where the singularities of the ambient space are resolved. For the favorable CY hypersurface $X,{ }^{17}$ the harmonic $(1,1)$-forms for $H^{2}(X, \mathbb{Z}), J_{r}$ with $r=1,2, \cdots, h^{1,1}$, can be obtained by the pull-backs of $(1,1)$-forms on the ambient space. Then, the intersection numbers $d_{r s t}$ of $X$ can be calculated by

$$
d_{r s t}=\int_{X} J_{r} \wedge J_{s} \wedge J_{t}
$$

Here, the $J_{r}$ stand for the basis of the Picard group. Finally, we describe the Kähler cone of $X$, determining the allowed Kähler moduli $t^{r}$. For the favorable CY hypersurface, the Kähler cone of $X$ is also derived from that of the ambient space whose Kähler cone is calculated by the data of polytope. When we expand the (1,1)-form $J$ as $J=t^{r} J_{r}$, the Kähler moduli $t^{r}$ satisfy

$$
\sum_{r} K_{r}^{\hat{r}} t^{r} \geq 0
$$

where we introduce the Kähler cone matrix $K=\left[K_{r}^{\hat{r}}\right]$ and $\hat{r}$ runs over the facets of the Kähler cone. Note that the two examples we analyze later have a few different triangulation corresponding to a partial resolution for the ambient toric varieties. In that case, the Kähler cone is given by the union of several sub-cones:

$$
K=\bigcup_{j} K_{j}
$$

Among them, only 16 manifolds have a non-trivial fundamental group which is an important ingredient in introducing Wilson-lines on quotient CY threefolds [71]. We focus on these particular class of CY threefolds in the context of heterotic string theory with standard embedding. ${ }^{18}$ For illustrative purposes, we deal with five quotient CY threefolds. (For more details about the toric data as well as topological data of CY threefolds, we refer to ref. [72].) In particular, we focus on the Kähler moduli sector by assuming that the complex structure moduli sector is stabilized by some mechanism at a high scale.

1. The first example of CY threefold with non-trivial fundamental group has $h^{1,1}=3$ Kähler moduli, $h^{2,1}=59$ complex structure moduli, i.e., Euler number $\chi=-112$ (corresponding to the "downstairs" CY threefold $X_{14}$ in ref. [72]). We will focus on

\footnotetext{
${ }^{17}$ The favorable CY means that the hodge number $h^{1,1}$ of $X$ is the same with that of the ambient space.

${ }^{18}$ The heterotic string models in the context of non-standard embedding are discussed on such a particular class of CY threefolds [72].
} 
the Kähler moduli sector since the complex structure moduli sector is complicated to analyze. The prepotential in the Kähler moduli sector is given by

$$
G=t^{1} t^{2} t^{3},
$$

with non-vanishing triple intersection number $\kappa_{123}=1$. The Kähler cone is given by $K=\operatorname{diag}(1,1,1)$. Hence, the superpotential of matter fields associated with the Kähler moduli has the same functional form as one in the example of section 3.2,

$$
W=A^{1} A^{2} A^{3} .
$$

Hence, in addition to $\Pi_{a=1}^{3} \mathrm{SL}(2, \mathbb{Z})_{a}$, there exists the $S_{4}$ flavor symmetry, which can be enhanced to $S_{4} \rtimes \mathbb{Z}_{2}^{C P}$ symmetry, taking into account the CP transformation. The symmetry is then embedded into the generalized symplectic $\operatorname{Sp}(8, \mathbb{Z})$ flavor symmetry in the Kähler moduli space.

2. The second example of three Kähler parameters of CY has $h^{2,1}=59$ complex structure moduli, i.e., Euler number $\chi=-112$ (corresponding to the downstairs CY threefold $X_{6}$ in ref. [72]). When we focus on the Kähler moduli sector, the prepotential is given by

$$
G=t^{1} t^{2} t^{3}-2 t^{2}\left(t^{3}\right)^{2}
$$

with non-vanishing triple intersection numbers $\kappa_{123}=1$ and $\kappa_{233}=-4$. Here, the intersection number is negative, but it is possible to redefine the modulus field as $\widetilde{t}^{1} \equiv t^{1}-2 t^{3}$, corresponding to the Kähler cone $K=\operatorname{diag}(1,1,1)$. It provides the superpotential of matter fields associated with the Kähler moduli,

$$
W=A^{1} A^{2} A^{3}-2 A^{2}\left(A^{3}\right)^{2},
$$

which has a flavor structure similar to the first example of CY. Indeed, when we redefine the matter fields as $\widetilde{A}^{1} \equiv A^{1}-2 A^{3}$, the matter superpotential reduces to

$$
W=\widetilde{A}^{1} A^{2} A^{3} \text {. }
$$

Hence, we find that $S_{4}$ flavor symmetry is realized on this class of CY threefold and its enhanced $S_{4} \rtimes \mathbb{Z}_{2}^{C P}$ symmetry together with the CP transformation can be embedded into the generalized symplectic $\operatorname{Sp}(8, \mathbb{Z})$ flavor symmetry.

3. The third example of three Kähler parameters of CY has $h^{2,1}=43$ complex structure moduli, i.e., Euler number $\chi=-80$ (corresponding to the downstairs CY threefold $X_{5}$ in ref. [72]). The prepotential in the Kähler moduli sector is given by

$$
G=t^{1} t^{2} t^{3}-2 t^{1}\left(t^{3}\right)^{2}+2 t^{2}\left(t^{3}\right)^{2}-8\left(t^{3}\right)^{3},
$$

with non-vanishing triple intersection numbers $\kappa_{123}=1, \kappa_{133}=-4, \kappa_{233}=4$ and $\kappa_{333}=-48$. Here, the Kähler cone is given by

$$
K=\left(\begin{array}{ccc}
0 & 1 & -2 \\
1 & 0 & 0 \\
0 & 0 & 1
\end{array}\right),
$$


constraining the Kähler moduli to satisfy eq. (3.61). It provides the superpotential of matter fields associated with the Kähler moduli,

$$
W=A^{1} A^{2} A^{3}-2 A^{1}\left(A^{3}\right)^{2}+2 A^{2}\left(A^{3}\right)^{2}-8\left(A^{3}\right)^{3} .
$$

There is the overall modular symmetry $\operatorname{SL}(2, \mathbb{Z})_{\text {overall }}$. It seems that other non-trivial flavor symmetries are absent in this system. There is $\mathbb{Z}_{2}$ symmetry such as

$$
A^{1} \rightarrow A^{2}, \quad A^{2} \rightarrow A^{1}, \quad A^{3} \rightarrow-A^{3},
$$

up to $\mathrm{U}(1)_{R}$ symmetry $W \rightarrow-W$.

4. The fourth example of three Kähler parameters of CY has $h^{2,1}=75$ complex structure moduli, i.e., Euler number $\chi=-144$ (corresponding to the downstairs CY threefold $X_{7}$ in ref. [72]). The prepotential in the Kähler moduli sector is given by

$$
G=t^{1} t^{2} t^{3}-2\left(t^{2}\right)^{2} t^{3}-2 t^{1}\left(t^{3}\right)^{2}+8\left(t^{3}\right)^{2}
$$

with non-vanishing triple intersection numbers $\kappa_{123}=1, \kappa_{223}=\kappa_{133}=-4$ and $\kappa_{333}=48$. Here, the Kähler cone is given by

$$
K=\left(\begin{array}{ccc}
1 & -2 & 0 \\
0 & 0 & 1 \\
0 & 1 & -2
\end{array}\right),
$$

constraining the Kähler moduli to satisfy eq. (3.61). It provides the superpotential of matter fields associated with the Kähler moduli,

$$
W=A^{1} A^{2} A^{3}-2\left(A^{2}\right)^{2} A^{3}-2 A^{1}\left(A^{3}\right)^{2}+8\left(A^{3}\right)^{2} .
$$

There is the overall modular symmetry $\mathrm{SL}(2, \mathbb{Z})_{\text {overall, }}$ but other non-trivial flavor symmetries are absent in this system.

5. The last example of three Kähler parameters of CY has $h^{2,1}=75$ complex structure moduli, i.e., Euler number $\chi=-144$ (corresponding to the downstairs CY threefold $X_{8}$ in ref. [72]). The prepotential in the Kähler moduli sector is given by

$$
G=t^{1} t^{2} t^{3}-2 t^{1}\left(t^{3}\right)^{2}-2 t^{2}\left(t^{3}\right)^{2}+8\left(t^{3}\right)^{2},
$$

with non-vanishing triple intersection numbers $\kappa_{123}=1, \kappa_{133}=\kappa_{233}=-4$ and $\kappa_{333}=48$. Here, the Kähler cone is given by

$$
K=\left(\begin{array}{ccc}
0 & 0 & 1 \\
1 & 0 & -2 \\
0 & 1 & -2
\end{array}\right),
$$

constraining the Kähler moduli to satisfy eq. (3.61). It provides the superpotential of matter fields associated with the Kähler moduli,

$$
W=A^{1} A^{2} A^{3}-2 A^{1}\left(A^{3}\right)^{2}-2 A^{2}\left(A^{3}\right)^{2}+8\left(A^{3}\right)^{2} .
$$


There is the overall modular symmetry $\mathrm{SL}(2, \mathbb{Z})_{\text {overall. }}$ In addition, there exists a $\mathbb{Z}_{2} \simeq S_{2}$ flavor symmetry between $A^{1}$ and $A^{2}$ and it is further enhanced to $\mathbb{Z}_{2} \rtimes \mathbb{Z}_{2}^{C P}$ symmetry, taking into account the CP transformation. Since the $\mathbb{Z}_{2}$ transformation is the same with $\mathcal{P}_{12}$ in eq. (3.51), the symmetry is then embedded into the $\operatorname{Sp}(8, \mathbb{Z})$ generalized symplectic flavor symmetry.

We find that non-trivial flavor symmetries on three-parameter examples of CY threefolds are given by $S_{4}$ and $\mathbb{Z}_{2}$ symmetries, enlarging to $S_{4} \rtimes \mathbb{Z}_{2}^{\mathrm{CP}}$ and $\mathbb{Z}_{2} \rtimes \mathbb{Z}_{2}^{\mathrm{CP}}$ taking into account the $\mathbb{Z}_{2}^{\mathrm{CP}}$ symmetry. Hence, the $S_{4}$ flavor symmetry appears in not only the exact orbifold limit but also a certain class of CY threefolds such as the first and second examples. In addition, all examples enjoy the overall modular symmetry $\operatorname{SL}(2, \mathbb{Z})_{\text {overall }}$, which looks $\mathbb{Z}_{3}$ symmetry, $A^{i} \rightarrow e^{2 \pi i / 3} A^{i}$ in the field-theoretical viewpoint. The above $S_{4}$ and $\mathbb{Z}_{2}$ symmetries are commutable with this $\mathbb{Z}_{3}$ symmetry.

Our analysis is limited to specific CY threefolds, but it is interesting to examine the flavor symmetries on a more broad class of CY threefolds. We leave it for future work.

\section{Conclusions and discussions}

In this paper, we examined the origin of flavor symmetry in the context of heterotic string theory on CY threefolds, including toroidal orbifolds. The flavor structure of matter zeromodes in the context of heterotic string theory with standard embedding is governed by the geometrical structure of CY threefolds because the matter and moduli fields are in one-to-one correspondence with each other. It indicates that the flavor structure is closely related to the symplectic structure of CY moduli spaces. Furthermore, the $4 \mathrm{D} \mathrm{CP}$ and the $\mathrm{U}(1)_{R}$ symmetries were regarded as the outer automorphism of the symplectic modular group [23] and the rotation of holomorphic three-form of CY threefolds [29], respectively. These observations motivate us to thoroughly investigate the relation among flavor, CP, and $\mathrm{U}(1)_{R}$ symmetries on the basis of $4 \mathrm{D}$ effective action. This approach would yield the ultra-violet origin of models with modular flavor symmetry in the bottom-up approach.

In section 2 , we gave a unified picture of these flavor, $\mathrm{CP}$, and $\mathrm{U}(1)_{R}$ symmetries from the viewpoint of generalized symplectic symmetry $G \mathrm{Sp}(2 h+2, \mathbb{C}) \simeq \operatorname{Sp}(2 h+2, \mathbb{C}) \rtimes \mathbb{Z}_{2}^{\mathrm{CP}} \times$ $\mathrm{U}(1)_{R}$, with $h$ being the number of either complex structure moduli or Kähler moduli. It was known that the traditional flavor symmetry was reflected by the flavor structure of Yukawa couplings, but our results suggest that the flavor symmetry $G_{\text {flavor }}$ is enlarged to some non-Abelian groups $G_{\text {flavor }} \rtimes \mathbb{Z}_{2}^{\mathrm{CP}}$ by the CP symmetry on a general class of CY threefolds with standard embedding. To exemplify these results, we deal with toroidal orbifolds with and without resolutions, and three-parameter examples of CY threefolds. It turned out that three untwisted modes associated with three Kähler moduli enjoy $S_{4}$ flavor symmetry on toroidal orbifolds. On the $T^{6} / \mathbb{Z}_{3}$ and $T^{6} /\left(\mathbb{Z}_{3} \times \mathbb{Z}_{3}^{\prime}\right)$ orbifolds with small resolutions, there exist $\left(T^{\prime}\right)^{3}$ and $S_{3}$ symmetries among twisted modes, respectively. On the geometrical regime of $T^{6} /\left(\mathbb{Z}_{3} \times \mathbb{Z}_{3}^{\prime}\right)$ orbifold where the size of blow-up modes is larger than the string length, we find the $S_{9}$ flavor symmetry among blow-up models (corresponding to twisted modes in the blow-down limit). The three-parameter examples of CY threefolds 
also enjoy $\mathbb{Z}_{2}$ and $S_{4}$ flavor symmetries as discussed in section 3.5. Hence, non-trivial flavor symmetries such as $S_{4}$ appear in not only the exact orbifold limit but also a certain class of CY threefolds. All the flavor symmetries we analyzed have an origin in the symplectic modular symmetry and are enlarged to non-Abelian groups by the CP symmetry.

In this paper, we have proposed the unification of flavor, $\mathrm{CP}$, and $\mathrm{U}(1)_{R}$ symmetries originated from the symplectic modular symmetry, and exemplify the flavor symmetries on CY threefolds, including toroidal results. However, there exist several future directions to pursue.

- Yukawa couplings on CY threefolds

On the toroidal backgrounds, moduli-dependent Yukawa couplings are estimated by the modular weight of matter fields, and their functional form is described by a certain modular form of $\mathrm{SL}(2, \mathbb{Z})$ symmetry. On the other hand, the results of section 3.5 exhibit the existence of $S_{4}$ flavor symmetry of matter fields on some CY threefolds equipping the effective $\mathrm{SL}(2, \mathbb{Z})$ modular symmetry. Hence, the Yukawa couplings including quantum corrections would be described by the modular form of $S_{4}$ flavor symmetry, controlled by the underlying symplectic modular symmetry. In this paper, we have focused on the CY moduli spaces at the classical level, but loop and instanton corrections calculated in the topological string theory appear in the prepotential as well as Yukawa couplings [73, 74]. Also, massive modes generate the higher-order couplings, which are described in an appropriate derivative of the prepotential [75]. It would be fascinating to figure out the flavor structure of matter fields, including these corrections. We hope to report on this interesting direction in the future.

- Spontaneous breaking of flavor symmetry

From the results in sections 3.3 and 3.4, there the $S_{3}$ and $S_{9}$ flavor symmetries of twisted modes on $T^{6} /\left(\mathbb{Z}_{3} \times \mathbb{Z}_{3}^{\prime}\right)$ orbifold with small resolutions and its geometrical regime, respectively. It indicates the spontaneous breaking of flavor symmetry would be induced by the blow-up modes. To understand the underlying structure, it is required to interpolate both the orbifold and geometrical regime. Including the stabilization of blow-up modes, we leave this topic for future research.

- Modular symmetric behavior of the twisted modes

In the limit that the size of blow-up moduli is sufficiently large, 9 twisted modes in the example of section 3.4 have $S_{9}$ flavor symmetry in $\operatorname{Sp}(18, \mathbb{Z})$ modular symmetry. Similarly, 27 twisted modes in the example of section 3.2 may also have the $S_{27}$ flavor symmetry in $\operatorname{Sp}(54, \mathbb{Z})$ modular symmetry. On the other hand, the symmetry $S_{27}$ as well as $\operatorname{Sp}(54, \mathbb{Z})$ loses the geometrically meaning in the blow-down orbifold limit. However, twisted modes transform non-trivially under the $\mathrm{SL}(2, \mathbb{Z})$ modular symmetry of untwisted modes because of the structure of prepotential and Kähler potential. That is a quite trivial transition of modular symmetric behavior of the twisted modes. In this paper, we have understood just two regimes, i.e., sufficiently large blow-up moduli regime $\left(\operatorname{Im}\left(v^{r}\right) \gg 1\right)$ and the blow-down orbifold limit $\left(\left|c^{r}\right| \ll 1\right)$ in the context of symplectic modular symmetry. The middle regime is unclear in our analysis. 
It was conjectured by ref. [76] that the blow-up moduli in both regimes would be related by $c^{r} \simeq \exp \left(2 \pi i v^{r}\right)$. It is interesting to find out a similar relation of twisted matters, which brings us to understand the whole picture of modular symmetric behavior.

\section{Acknowledgments}

The authors would like to thank Hiroshi Ohki for useful discussions, and especially to the anonymous referee for a careful reading of the manuscript and constructive suggestions. H.O. was supported in part by JSPS KAKENHI Grant Numbers JP19J00664 and JP20K14477.

\section{A Modular form of weight 1 for $T^{\prime}$}

Following ref. [58], we define the following functions,

$$
\hat{e}_{1}(\tau)=\frac{\eta^{3}(3 \tau)}{\eta(\tau)}, \quad \hat{e}_{2}(\tau)=\frac{\eta^{3}(\tau / 3)}{\eta(\tau)},
$$

by using the Dedekind eta function $\eta(\tau)$,

$$
\eta(\tau)=q^{1 / 24} \prod_{n=1}^{\infty}\left(1-q^{n}\right),
$$

with $q=e^{2 \pi i \tau}$. Under the $T$-transformation, they transform

$$
\hat{e}_{1} \rightarrow \omega \hat{e}_{1}, \quad \hat{e}_{2} \rightarrow 3(1-\omega) \hat{e}_{1}+\hat{e}_{2},
$$

with $w=e^{2 \pi i / 3}$. Under the $S$-transformation, they transform

$$
\hat{e}_{1} \rightarrow 3^{-3 / 2} i(-\tau) \hat{e}_{2}, \quad \hat{e}_{2} \rightarrow 3^{3 / 2} i(-\tau) \hat{e}_{1} .
$$

By using these functions, we can construct the doublet of $T^{\prime}$, which transforms under the $T$-transformation,

$$
\left(\begin{array}{l}
x_{1} \\
y_{1}
\end{array}\right) \rightarrow \rho(T)\left(\begin{array}{l}
x_{1} \\
y_{1}
\end{array}\right),
$$

and under the $S$-transformation

$$
\left(\begin{array}{l}
x_{1} \\
y_{1}
\end{array}\right) \rightarrow(-\tau) \rho(S)\left(\begin{array}{l}
x_{1} \\
y_{1}
\end{array}\right)
$$

where

$$
\rho(T)=\left(\begin{array}{ll}
\omega & 0 \\
0 & 1
\end{array}\right), \quad \rho(S)=-\frac{i}{\sqrt{3}}\left(\begin{array}{cc}
1 & \sqrt{2} \\
\sqrt{2} & -1
\end{array}\right) .
$$

We follow the representation basis in refs. [47, 48], which is different from one in ref. [58]. 
Explicitly, the modular form of weight 1 for the $T^{\prime}$ doublet can be written by

$$
\left(\begin{array}{c}
x_{1} \\
y_{1}
\end{array}\right)=\left(\begin{array}{c}
-\sqrt{2} \hat{e}_{1} \\
\hat{e}_{1}+\frac{1}{3} \hat{e}_{2}
\end{array}\right) .
$$

Their $q$-expansions can be written by

$$
\begin{array}{r}
x_{1}=-\sqrt{2} q^{1 / 3}+\cdots, \\
y_{1}=1 / 3+\cdots .
\end{array}
$$

Open Access. This article is distributed under the terms of the Creative Commons Attribution License (CC-BY 4.0), which permits any use, distribution and reproduction in any medium, provided the original author(s) and source are credited.

\section{References}

[1] F. Feruglio, Are neutrino masses modular forms?, arXiv:1706.08749 [INSPIRE].

[2] T. Kobayashi, K. Tanaka and T.H. Tatsuishi, Neutrino mixing from finite modular groups, Phys. Rev. D 98 (2018) 016004 [arXiv:1803.10391] [INSPIRE].

[3] J.T. Penedo and S.T. Petcov, Lepton masses and mixing from modular $S_{4}$ symmetry, Nucl. Phys. B 939 (2019) 292 [arXiv:1806.11040] [INSPIRE].

[4] J.C. Criado and F. Feruglio, Modular invariance faces precision neutrino data, SciPost Phys. 5 (2018) 042 [arXiv: 1807.01125] [INSPIRE].

[5] T. Kobayashi, N. Omoto, Y. Shimizu, K. Takagi, M. Tanimoto and T.H. Tatsuishi, Modular $A_{4}$ invariance and neutrino mixing, JHEP 11 (2018) 196 [arXiv:1808.03012] [INSPIRE].

[6] P.P. Novichkov, J.T. Penedo, S.T. Petcov and A.V. Titov, Modular $S_{4}$ models of lepton masses and mixing, JHEP 04 (2019) 005 [arXiv: 1811.04933] [INSPIRE].

[7] P.P. Novichkov, J.T. Penedo, S.T. Petcov and A.V. Titov, Modular $A_{5}$ symmetry for flavour model building, JHEP 04 (2019) 174 [arXiv: 1812.02158] [INSPIRE].

[8] F.J. de Anda, S.F. King and E. Perdomo, SU(5) grand unified theory with $A_{4}$ modular symmetry, Phys. Rev. D 101 (2020) 015028 [arXiv:1812.05620] [InSPIRE].

[9] H. Okada and M. Tanimoto, CP violation of quarks in $A_{4}$ modular invariance, Phys. Lett. $B$ 791 (2019) 54 [arXiv:1812.09677] [INSPIRE].

[10] T. Kobayashi, Y. Shimizu, K. Takagi, M. Tanimoto, T.H. Tatsuishi and H. Uchida, Finite modular subgroups for fermion mass matrices and baryon/lepton number violation, Phys. Lett. B 794 (2019) 114 [arXiv: 1812.11072] [INSPIRE].

[11] P.P. Novichkov, S.T. Petcov and M. Tanimoto, Trimaximal neutrino mixing from modular $A_{4}$ invariance with residual symmetries, Phys. Lett. B 793 (2019) 247 [arXiv:1812.11289] [INSPIRE].

[12] R. de Adelhart Toorop, F. Feruglio and C. Hagedorn, Finite modular groups and lepton mixing, Nucl. Phys. B 858 (2012) 437 [arXiv:1112.1340] [INSPIRE].

[13] H.P. Nilles, M. Ratz, A. Trautner and P.K.S. Vaudrevange, $\mathcal{C P}$ violation from string theory, Phys. Lett. B 786 (2018) 283 [arXiv: 1808.07060] [INSPIRE]. 
[14] P.P. Novichkov, J.T. Penedo, S.T. Petcov and A.V. Titov, Generalised CP symmetry in modular-invariant models of Flavour, JHEP 07 (2019) 165 [arXiv: 1905.11970] [INSPIRE].

[15] S. Ferrara, D. Lüst, A.D. Shapere and S. Theisen, Modular invariance in supersymmetric field theories, Phys. Lett. B 225 (1989) 363 [INSPIRE].

[16] S. Ferrara, .D. Lüst and S. Theisen, Target space modular invariance and low-energy couplings in orbifold compactifications, Phys. Lett. B 233 (1989) 147 [INSPIRE].

[17] J.P. Derendinger, S. Ferrara, C. Kounnas and F. Zwirner, On loop corrections to string effective field theories: Field dependent gauge couplings and sigma model anomalies, Nucl. Phys. B 372 (1992) 145 [INSPIRE].

[18] L.E. Ibáñez and D. Lüst, Duality anomaly cancellation, minimal string unification and the effective low-energy Lagrangian of $4 D$ strings, Nucl. Phys. B 382 (1992) 305 [hep-th/9202046] [INSPIRE].

[19] T. Kobayashi, S. Nagamoto and S. Uemura, Modular symmetry in magnetized/intersecting D-brane models, PTEP 2017 (2017) 023B02 [arXiv: 1608.06129] [INSPIRE].

[20] A. Baur, H.P. Nilles, A. Trautner and P.K.S. Vaudrevange, Unification of flavor, $C P$, and modular symmetries, Phys. Lett. B 795 (2019) 7 [arXiv:1901.03251] [INSPIRE].

[21] T. Kobayashi and H. Otsuka, Classification of discrete modular symmetries in Type IIB flux vacua, Phys. Rev. D 101 (2020) 106017 [arXiv:2001.07972] [INSPIRE].

[22] T. Kobayashi and H. Otsuka, Challenge for spontaneous CP violation in Type IIB orientifolds with fluxes, Phys. Rev. D 102 (2020) 026004 [arXiv: 2004.04518] [INSPIRE].

[23] K. Ishiguro, T. Kobayashi and H. Otsuka, Spontaneous CP-violation and symplectic modular symmetry in Calabi-Yau compactifications, Nucl. Phys. B 973 (2021) 115598 [arXiv:2010.10782] [INSPIRE].

[24] A. Strominger, Special geometry, Commun. Math. Phys. 133 (1990) 163 [INSPIRE].

[25] P. Candelas and X. de la Ossa, Moduli space of Calabi-Yau manifolds, Nucl. Phys. B 355 (1991) 455 [INSPIRE].

[26] G.-J. Ding, F. Feruglio and X.-G. Liu, Automorphic forms and fermion masses, JHEP 01 (2021) 037 [arXiv:2010.07952] [INSPIRE].

[27] A. Baur, M. Kade, H.P. Nilles, S. Ramos-Sanchez and P.K.S. Vaudrevange, Siegel modular flavor group and CP from string theory, Phys. Lett. B 816 (2021) 136176 [arXiv: 2012.09586] [INSPIRE].

[28] H.P. Nilles, S. Ramos-Sanchez, A. Trautner and P.K.S. Vaudrevange, Orbifolds from $\operatorname{Sp}(4, Z)$ and their modular symmetries, Nucl. Phys. B 971 (2021) 115534 [arXiv:2105.08078] [INSPIRE].

[29] E. Witten, Symmetry breaking patterns in superstring models, Nucl. Phys. B 258 (1985) 75.

[30] B. de Wit, F. Vanderseypen and A. Van Proeyen, Symmetry structure of special geometries, Nucl. Phys. B 400 (1993) 463 [hep-th/9210068] [InSPIRE].

[31] B. de Wit and A. Van Proeyen, Potentials and symmetries of general gauged $N=2$ supergravity: Yang-Mills models, Nucl. Phys. B 245 (1984) 89 [inSPIRE].

[32] D.J. Gross, J.A. Harvey, E.J. Martinec and R. Rohm, Heterotic string theory. 1. The free heterotic string, Nucl. Phys. B 256 (1985) 253 [inSPIRE]. 
[33] D.J. Gross, J.A. Harvey, E.J. Martinec and R. Rohm, Heterotic string theory. 2. The interacting heterotic string, Nucl. Phys. B 267 (1986) 75 [InSPIRE].

[34] N. Seiberg, Observations on the moduli space of superconformal field theories, Nucl. Phys. B 303 (1988) 286 [INSPIRE].

[35] S. Cecotti, S. Ferrara and L. Girardello, Geometry of type II superstrings and the moduli of superconformal field theories, Int. J. Mod. Phys. A 4 (1989) 2475.

[36] L.J. Dixon, V. Kaplunovsky and J. Louis, On effective field theories describing $(2,2)$ vacua of the heterotic string, Nucl. Phys. B 329 (1990) 27 [INSPIRE].

[37] M. Billó, R. D'Auria, S. Ferrara, P. Fré, P. Soriani and A. Van Proeyen, R symmetry and the topological twist of $N=2$ effective supergravities of heterotic strings, Int. J. Mod. Phys. A 12 (1997) 379 [hep-th/9505123] [INSPIRE].

[38] T. Araki, K.-S. Choi, T. Kobayashi, J. Kubo and H. Ohki, Discrete R-symmetry anomalies in heterotic orbifold models, Phys. Rev. D 76 (2007) 066006 [arXiv:0705.3075] [InSPIRE].

[39] T. Araki, T. Kobayashi, J. Kubo, S. Ramos-Sanchez, M. Ratz and P.K.S. Vaudrevange, (Non-)Abelian discrete anomalies, Nucl. Phys. B 805 (2008) 124 [arXiv:0805.0207] [INSPIRE].

[40] A. Strominger and E. Witten, New manifolds for superstring compactification, Commun. Math. Phys. 101 (1985) 341 [InSPIRE].

[41] M. Dine, R.G. Leigh and D.A. MacIntire, Of CP and other gauge symmetries in string theory, Phys. Rev. Lett. 69 (1992) 2030 [hep-th/9205011] [INSPIRE].

[42] K.-w. Choi, D.B. Kaplan and A.E. Nelson, Is CP a gauge symmetry?, Nucl. Phys. B 391 (1993) 515 [hep-ph/9205202] [INSPIRE].

[43] A. Strominger, Topology of superstring compactification, NSF-ITP-85-109 (1985).

[44] F. Feruglio, C. Hagedorn and R. Ziegler, Lepton mixing parameters from discrete and $C P$ symmetries, JHEP 07 (2013) 027 [arXiv:1211.5560] [INSPIRE].

[45] M. Holthausen, M. Lindner and M.A. Schmidt, CP and discrete flavour symmetries, JHEP 04 (2013) 122 [arXiv: 1211.6953] [INSPIRE].

[46] M.-C. Chen, M. Fallbacher, K.T. Mahanthappa, M. Ratz and A. Trautner, CP violation from finite groups, Nucl. Phys. B 883 (2014) 267 [arXiv: 1402.0507] [INSPIRE].

[47] H. Ishimori, T. Kobayashi, H. Ohki, Y. Shimizu, H. Okada and M. Tanimoto, Non-Abelian discrete symmetries in particle physics, Prog. Theor. Phys. Suppl. 183 (2010) 1 [arXiv: 1003.3552] [INSPIRE].

[48] H. Ishimori, T. Kobayashi, H. Ohki, H. Okada, Y. Shimizu and M. Tanimoto, An introduction to non-Abelian discrete symmetries for particle physicists, Lecture Notes in Physics colume 858, Springer, Germany (2012) [INSPIRE].

[49] D. Shevitz, The global structure of a $c=9$ type $(2,2)$ moduli space, Nucl. Phys. B 338 (1990) 283 [INSPIRE].

[50] B.L. Rachlin and T.W. Kephart, Spontaneous breaking of gauge groups to discrete symmetries, JHEP 08 (2017) 110 [arXiv: 1702.08073] [INSPIRE].

[51] K. Ishiguro and H. Otsuka, Sharpening the boundaries between flux landscape and swampland by tadpole charge, JHEP 12 (2021) 017 [arXiv:2104.15030] [INSPIRE]. 
[52] S. Hamidi and C. Vafa, Interactions on orbifolds, Nucl. Phys. B 279 (1987) 465 [InSPIRE].

[53] L.J. Dixon, D. Friedan, E.J. Martinec and S.H. Shenker, The conformal field theory of orbifolds, Nucl. Phys. B 282 (1987) 13 [INSPIRE].

[54] T.T. Burwick, R.K. Kaiser and H.F. Muller, General Yukawa couplings of strings on $Z(N)$ orbifolds, Nucl. Phys. B 355 (1991) 689.

[55] W. Lerche, D. Lüst and N.P. Warner, Duality symmetries in $N=2$ Landau-Ginzburg models, Phys. Lett. B 231 (1989) 417 [INSPIRE].

[56] J. Lauer, J. Mas and H.P. Nilles, Duality and the Role of Nonperturbative Effects on the World Sheet, Phys. Lett. B 226 (1989) 251 [INSPIRE].

[57] J. Lauer, J. Mas and H.P. Nilles, Twisted sector representations of discrete background symmetries for two-dimensional orbifolds, Nucl. Phys. B 351 (1991) 353 [inSPIRE].

[58] X.-G. Liu and G.-J. Ding, Neutrino masses and mixing from double covering of finite modular groups, JHEP 08 (2019) 134 [arXiv:1907.01488] [INSPIRE].

[59] T. Kobayashi, S. Nagamoto, S. Takada, S. Tamba and T.H. Tatsuishi, Modular symmetry and non-Abelian discrete flavor symmetries in string compactification, Phys. Rev. D 97 (2018) 116002 [arXiv: 1804.06644] [INSPIRE].

[60] T. Kobayashi and S. Tamba, Modular forms of finite modular subgroups from magnetized D-brane models, Phys. Rev. D 99 (2019) 046001 [arXiv:1811.11384] [InSPIRE].

[61] H. Ohki, S. Uemura and R. Watanabe, Modular flavor symmetry on a magnetized torus, Phys. Rev. D 102 (2020) 085008 [arXiv:2003.04174] [INSPIRE].

[62] S. Kikuchi, T. Kobayashi, S. Takada, T.H. Tatsuishi and H. Uchida, Revisiting modular symmetry in magnetized torus and orbifold compactifications, Phys. Rev. D 102 (2020) 105010 [arXiv : 2005.12642] [INSPIRE].

[63] S. Kikuchi, T. Kobayashi, H. Otsuka, S. Takada and H. Uchida, Modular symmetry by orbifolding magnetized $T^{2} \times T^{2}$ : realization of double cover of $\Gamma_{N}$, JHEP 11 (2020) 101 [arXiv: 2007.06188] [INSPIRE].

[64] S. Kikuchi, T. Kobayashi and H. Uchida, Modular flavor symmetries of three-generation modes on magnetized toroidal orbifolds, Phys. Rev. D 104 (2021) 065008 [arXiv:2101.00826] [INSPIRE].

[65] Y. Almumin, M.-C. Chen, V. Knapp-Pérez, S. Ramos-Sánchez, M. Ratz and S. Shukla, Metaplectic flavor symmetries from magnetized tori, JHEP 05 (2021) 078 [arXiv: 2102.11286] [INSPIRE].

[66] Y. Tatsuta, Modular symmetry and zeros in magnetic compactifications, JHEP 10 (2021) 054 [arXiv: 2104.03855] [INSPIRE].

[67] M. Cvetic, B.A. Ovrut and J. Louis, The Zamolodchikov metric and effective Lagrangians in string theory, Phys. Rev. D 40 (1989) 684.

[68] K. Ishiguro, T. Kobayashi and H. Otsuka, Hierarchical structure of physical Yukawa couplings from matter field Kähler metric, JHEP 07 (2021) 064 [arXiv:2103.10240] [INSPIRE].

[69] O. DeWolfe, A. Giryavets, S. Kachru and W. Taylor, Type IIA moduli stabilization, JHEP 07 (2005) 066 [hep-th/0505160] [INSPIRE].

[70] M. Kreuzer and H. Skarke, Complete classification of reflexive polyhedra in four-dimensions, Adv. Theor. Math. Phys. 4 (2000) 1209 [hep-th/0002240] [INSPIRE]. 
[71] V. Batyrev and M. Kreuzer, Integral cohomology and mirror symmetry for Calabi-Yau 3-folds, math/0505432 [INSPIRE].

[72] Y.-H. He, S.-J. Lee, A. Lukas and C. Sun, Heterotic model building: 16 special manifolds, JHEP 06 (2014) 077 [arXiv: 1309.0223] [INSPIRE].

[73] R. Gopakumar and C. Vafa, M theory and topological strings. 1, hep-th/9809187 [INSPIRE].

[74] R. Gopakumar and C. Vafa, M theory and topological strings. 2, hep-th/9812127 [INSPIRE].

[75] M. Bershadsky, S. Cecotti, H. Ooguri and C. Vafa, Kodaira-Spencer theory of gravity and exact results for quantum string amplitudes, Commun. Math. Phys. 165 (1994) 311 [hep-th/9309140] [INSPIRE].

[76] S. Groot Nibbelink, J. Held, F. Ruehle, M. Trapletti and P.K.S. Vaudrevange, Heterotic Z(6-II) MSSM orbifolds in blowup, JHEP 03 (2009) 005 [arXiv:0901.3059] [INSPIRE]. 